\title{
Analyse de l'activité de lycéens en situations pédagogiques de travail en groupe
}

Analysis of the activity of high school students in educational situations of group work

\section{Sylvain Connac et Carmen Rusu}

\section{(2) OpenEdition}

\section{Journals}

Édition électronique

URL : https://journals.openedition.org/activites/6705

DOI : 10.4000/activites.6705

ISSN : $1765-2723$

\section{Éditeur}

ARPACT - Association Recherches et Pratiques sur les ACTivités

\section{Référence électronique}

Sylvain Connac et Carmen Rusu, « Analyse de l'activité de lycéens en situations pédagogiques de travail en groupe », Activités [En ligne], 18-2 | 2021, mis en ligne le 15 octobre 2021, consulté le 07 avril 2022. URL : http://journals.openedition.org/activites/6705; DOI : https://doi.org/10.4000/ activites. 6705

Ce document a été généré automatiquement le 7 avril 2022.

\section{cc) (†)}

Activités est mis à disposition selon les termes de la licence Creative Commons Attribution - Pas d'Utilisation Commerciale - Pas de Modification 4.0 International. 


\title{
Analyse de l'activité de lycéens en situations pédagogiques de travail en groupe
}

Analysis of the activity of high school students in educational situations of group work

\author{
Sylvain Connac et Carmen Rusu
}

\section{NOTE DE L'ÉDITEUR}

Article soumis le 01/08/2020, accepté le 19/04/2021

\section{Introduction}

1 En lycée comme ailleurs, la difficile question de la prise en compte de la diversité des élèves est soulevée. Les classes ne peuvent qu'être hétérogènes, avec une double variable (Lorcerie, 2020) : celle des habiletés cognitives (qui rend les niveaux des élèves disparates) et celle de la dimension sociale (avec des élèves de statuts sociaux et ethniques différents, notamment pour celles et ceux issus de milieux défavorisés). Les pratiques pédagogiques de mise au travail des élèves ne pourront jamais résoudre l'ensemble de ces défis. Elles peuvent cependant s'organiser de telle sorte qu'elles s'accordent avec les logiques d'une différenciation pédagogique. Toutefois, plusieurs travaux récents relatifs à cette différenciation en présentent différentes limites, allant même jusqu'à aboutir à des conséquences inverses à celles annoncées: par de la différenciation, l'écart entre les élèves augmenterait, le niveau scolaire moyen baisserait et les plus fragiles se décourageraient (CNESCO, 2017 ; Kahn, 2010).

2 Sans chercher à renoncer à une évolution de ces effets délétères de la différenciation, d'autres approches existent pour tenter une prise en compte de l'hétérogénéité, sans 
risque de stigmatisation de la différence par une externalisation de l'aide ou une réduction des exigences. Nous allons explorer les pratiques collectives de coopération, à travers l'étude de travaux en groupe d'élèves, autour d'une situation-problème commune. Le principe pédagogique activé est celui de la recherche d'enrôlement (une des six postures d'étayage de J. Bruner, 2011), pour éviter d'avoir à « raccrocher » des élèves qui n'auraient pas " accroché » au démarrage d'une séquence d'enseignement et d'avoir recours à des stratégies de remédiation toujours très risquées parce qu'elles assignent un élève aux difficultés qu'il rencontre et se traduisent la plupart du temps par du renforcement sur un bas niveau taxonomique (Toullec-Théry, 2015). Nous allons donc étudier, à travers le compte-rendu de l'activité d'élèves, comment le travail en groupe peut les enrôler dans un processus de dévolution des problèmes, par l'intermédiaire de la formation de conflits socio-cognitifs.

\section{Le travail en groupe d'élèves au sein des pédagogies de la coopération - place du conflit socio-cognitif dans l'apprentissage}

3 Travailler en groupe est une activité courante pour un élève. Toutefois, cette pratique d'enseignement se justifie par des liens avec les processus d'apprentissages, principalement parce qu'elle est vectrice de conflits socio-cognitifs. Cette partie est aussi l'occasion de distinguer le travail en groupe d'autres modalités coopératives proches en apparence : le travail de groupe, le travail en équipe et le travail d'équipe.

\subsection{Travail en groupe et apprentissages}

4 Le travail en groupe (ou " travail en groupe d'apprentissage ») est une des déclinaisons pédagogiques de la coopération entre élèves. Celles-ci regroupent l'ensemble des situations où des élèves sont encouragés à produire ou à apprendre avec d'autres : au sein d'une action combinée, avec une intentionnalité et la possibilité reconnue par tous d'en tirer un bénéfice individuel (Connac, 2017b). Les principales déclinaisons coopératives sont l'aide, l'entraide, le tutorat, les marchés de connaissances, le travail en atelier, en groupe ou en équipe, les conseils coopératifs, les discussions démocratiques ou les jeux coopératifs.

5 Spécifique à ces organisations coopératives de l'activité scolaire, le travail en groupe correspond à une forme de mise en activité des élèves, à l'initiative d'un enseignant, qui s'appuie sur leurs représentations initiales pour les mettre à l'épreuve d'un obstacle cognitif $^{1}$ : « les contextes d'apprentissage les plus favorables sont avant tout influencés par les connaissances antérieures de l'élève » (Sander, Gris, Gvozdic, \& Scheibling-Sève, 2018, p. 59). Il est en mesure de participer au processus d'apprentissage en visant la majoration des représentations par la transition entre les deux premières phases de l'acte d'apprendre: de l'incompétence inconsciente à l'incompétence consciente (Connac, 2018b ; Favre, 2015). Ainsi, un travail en groupe peut susciter l'émergence de conflits cognitifs par l'exercice de conflits socio-cognitifs : la confrontation entre les connaissances premières des élèves (leurs représentations initiales) peut déclencher chez eux un litige cognitif, c'est-à-dire un désaccord entre eux pour conduire à un doute avec soi-même. C'est à partir de cette incertitude constatée, les conflits cognitifs exprimés par des questions que les élèves se posent, que les savoirs en jeu vont pouvoir 
être perçus comme des éléments de réponse (Meirieu, 1996). La transmission des savoirs scolaires, répondant à ces questions éprouvées par les élèves et conduisant à de nouvelles compréhensions, génèrerait alors des émotions positives (agréables) d'accomplissement, ce qui augmenterait l'intérêt et les efforts mis dans la tâche, ainsi qu'envers la qualité et la régulation des stratégies métacognitives d'apprentissage (Favre, 2015 ; Pekrun, 2006).

6 Le travail en groupe mobilise ainsi l'engagement des élèves pour susciter l'émergence de conflits cognitifs (suite à la production de conflits socio-cognitifs), la recherche de solutions, l'apparition de propositions inattendues et divergentes, le foisonnement des idées, la décentration des points de vue, le développement de capacités d'autoévaluation et de compétences langagières, l'engagement et, par l'étayage de l'enseignant, la clarification de la pensée (Blais \& Fleury, 1996). Les recherches sur les émotions (Audrin, 2020 ; Pekrun, 2014) soulignent l'impact qu'elles peuvent avoir sur les apprentissages : alors que des émotions négatives désactivantes comme l'ennui sont liées à un désinvestissement dans la tâche, les émotions négatives activantes (comme la frustration épistémique ou la confusion) peuvent conduire à vouloir éviter l'échec et ainsi à augmenter l'investissement dans la tâche. C'est en ce sens que les conflits cognitifs sont riches pour enseigner et pour apprendre.

\subsection{Travail en groupe et conflit socio-cognitif}

7 Le travail en groupe est une forme de coopération tournée vers l'étude d'une situationproblème (Rey \& Carette, 2019), pour que les élèves confrontent leurs stratégies de résolution et en viennent à douter de leurs certitudes pour s'ouvrir à de nouveaux apprentissages. "Dans la controverse avec autrui, il y a opposition entre deux centrations simultanées, celle du sujet et celle de son interlocuteur » (Rey \& Carette, 2019, p. 37). Le conflit socio-cognitif (la controverse avec autrui) est son objectif pédagogique prioritaire, même si d'autres intérêts émergent, dont la formation de processus de problématisation au cours desquels un élève met en question les conditions du problème parce qu'elles font défaut (Fabre \& Vellas, 2006).

Un conflit socio-cognitif correspond à une situation où des élèves, devant résoudre un problème identique, acceptent de confronter leurs avis pour explorer des idées et des arguments ignorés ou oubliés individuellement. «Un tel conflit survient entre les conceptions contradictoires de deux enfants (ou plus) rendues manifestes lors de leurs interactions communicationnelles » (Doise \& Mugny, 1981, p. 40). Il présente un intérêt lorsqu'il conduit à un conflit cognitif chez les sujets en interactions, c'est-à-dire lorsque la confrontation des idées génère une décentration quant à ses représentations et un doute quant à la solidité des conceptions initiales exprimées. Être confronté à un désaccord souligne que la notion n'est peut-être pas maitrisée et remet en question la compétence propre (Darnon, Buchs, \& Butera, 2006). Un conflit socio-cognitif se présente donc comme une stimulation intellectuelle par une déstabilisation cognitive (Buchs, Darnon, Quiamzade, Mugny, \& Butera, 2008). Autrement dit, son but n'est pas une conversation dialectique visant à établir un terrain d'entente entre des idées opposées, mais plutôt une conversation dialogique, pour que les partenaires, par la confrontation d'idées opposées, en viennent à approfondir leurs compréhensions mutuelles (Bakhtine, 1978). 
Ces controverses conduiraient à une augmentation de l'investissement individuel, à des bénéfices cognitifs, au développement de compétences interpersonnelles et à une curiosité épistémique, une motivation pour comprendre et acquérir de nouvelles connaissances (Buchs et al., 2008; Darnon et al., 2006). Un conflit socio-cognitif se présente ainsi comme une condition nécessaire à la décentration intellectuelle et préparant un terrain pour un rapport épistémique au savoir. "Les coordinations entre individus sont à l'origine des coordinations individuelles, elles les précèdent et les génèrent » (Doise \& Mugny, 1981, p. 34).

$10 \mathrm{Au}$ sein d'un travail en groupe, les élèves sont conduits à associer leurs actions et leurs attentions autour d'un même problème, ce qui les entraîne dans un processus de décentration et, potentiellement, dans un conflit entre leur point de vue et celui des partenaires. C'est ce conflit avec d'autres, autour d'une même tâche, qui génèrerait du doute, une peur de l'échec, de l'incertitude (un conflit cognitif) ainsi que des stratégies de tentatives pour réduire cette incertitude (Darnon et al., 2006). C'est cela qui incite à une restructuration cognitive :

«Si l'échange collectif peut certainement faciliter le travail cognitif et la formation des opérations, le conflit socio-cognitif peut, lui, dans certaines conditions et à un moment donné du développement de l'individu, les susciter. Certes, le conflit sociocognitif n'est pas en lui-même créateur de formes, mais il déclenche les déséquilibres qui rendent nécessaire cette élaboration» (Perret-Clermont, 1979, p. 206).

\subsection{Travail en groupe, de groupe, d'équipe ou en équipe?}

11 Pour distinguer les différentes formes de travail à plusieurs, nous utilisons la schématisation présentée dans la figure 1. Elle est organisée selon deux axes : celui des objectifs (de production ou d'apprentissage) et celui de la référence (externe ou interne - Favre, 2015).

Le travail en groupe se distingue du travail de groupe. Un travail de groupe est désigné comme une modalité de travail à plusieurs orientée vers la réalisation d'une tâche ou une production (le travail du groupe) plus que vers les apprentissages individuels par l'intermédiaire d'un conflit socio-cognitif (Connac, 2018a). En pédagogie, le travail de groupe est problématique parce qu'il priorise la tâche sur l'apprentissage, ce qui peut induire des malentendus chez certains élèves. Un travail en groupe se distingue aussi du travail d'équipe qui s'appuie davantage sur la cohésion entre les partenaires, mais priorise également une réalisation. Un travail en équipe concerne les formes de coopération relatives aux démarches de projets (Cottereau, 2007; Huber, 1999), principalement orientées vers les « éducations à " (Barthes, Lange, \& Tutiaux-Guillon, 2017). En s'y engageant à plusieurs, les élèves peuvent réinvestir des apprentissages et développer des compétences transversales (mener un projet à terme, rédiger des comptes-rendus, prendre la parole en public, soft-skills...). 
Figure 1 : Travail à plusieurs.

Figure 1: Working together

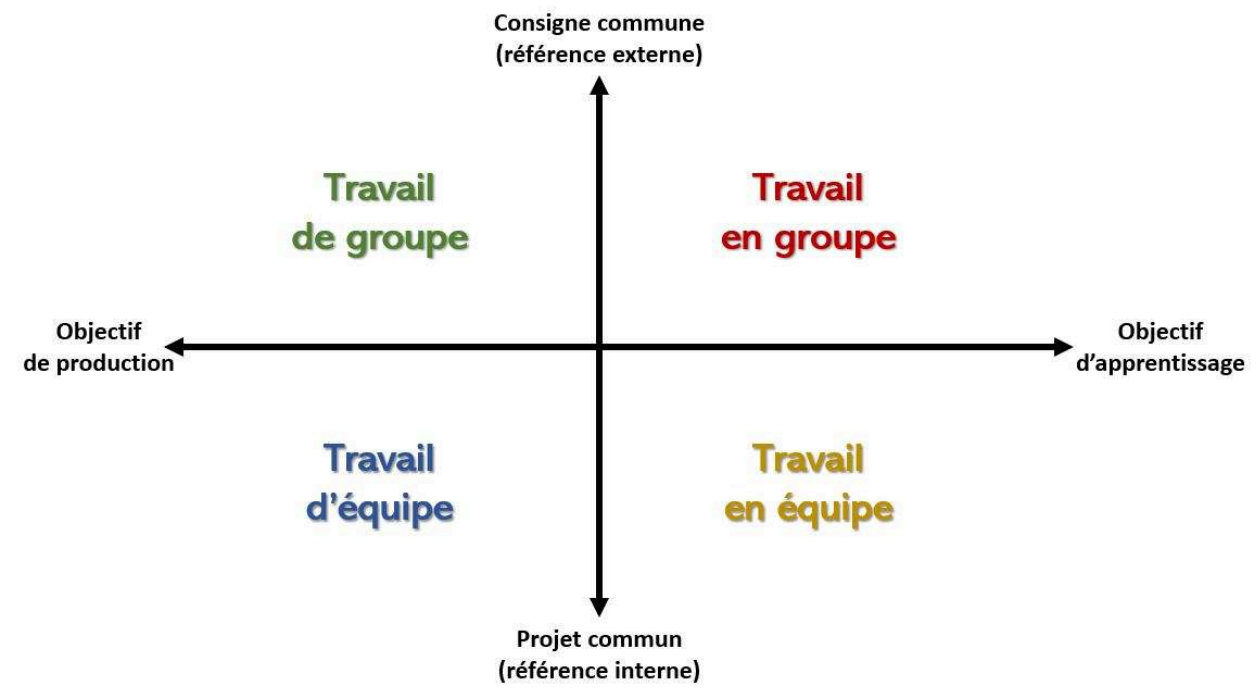

Le travail en équipe correspond donc à la forme coopérative des démarches de projet, au sein desquelles chaque co-équipier participe au projet de l'équipe. L'objectif prioritaire est de pousser les apprentissages jusqu'à en faire des compétences. La principale précaution est de s'assurer que chacun s'engage pleinement, en tire bénéfice sans une répartition inéquitable des tâches: à long terme, comme méthode d'enseignement, il est très important que tous les membres de l'équipe développent "une expérience efficace de travail en commun qui facilite à chaque membre l'acquisition de connaissances spécifiques à un domaine à partir de cet effort combiné " (Kirschner, Kirschner, Sweiller, \& Zambrano, 2018, p. 219). En pédagogie, un groupe diffère d'une équipe parce que le groupe cherche à valoriser la confrontation des idées pour ouvrir au questionnement ou à l'identification des talents individuels. Une équipe est plutôt tournée vers la recherche d'accords pour l'aboutissement d'un projet (même si des désaccords entre équipiers peuvent aider à améliorer la facture finale).

\subsection{Organisation d'un travail en groupe}

L'organisation d'un travail en groupe est une non-évidence. En effet, il ne suffit pas de mettre quatre élèves autour d'une table pour que l'activité soit cognitivement intéressante pour chacun d'eux. Darnon, Buchs et Butera (2006) expliquent que lorsque le conflit est relationnel, notamment par de la comparaison sociale, ou lorsqu'il est orienté vers un but de performance ( $v s$ but de maîtrise), il serait contreproductif du point de vue des apprentissages. «Le conflit n'a d'effets positifs que lorsqu'il est régulé de manière épistémique » (Darnon et al., 2006, p. 37).

De manière plus large, quatre formes de dérives ont pu être identifiées (Connac, 2018a) :

- attentionnelle : l'espace devient trop bruyant et trop agité pour faciliter la concentration ;

- fusionnelle: des élèves usent de stratégies dites de «consensus de complaisance » pour éviter les conflits d'idées ;

- productiviste : avec une confusion entre l'organisation coopérative - une partie de la tâche et les attendus d'apprentissages - l'activité espérée ; 
- différenciatrice: les élèves les plus avancés scolairement investissant les fonctions coopératives les plus mobilisatrices. inaccessibles individuellement, parce que les apports des partenaires et la coordination des connaissances réduisent la distance avec les informations ou la compréhension des problèmes à résoudre (Kirschner et al., 2018, p. 220). Cependant, il est apparu que tous les élèves n'ont pas besoin d'être en groupe pour travailler ou apprendre et que certaines circonstances de la coopération contribuent à interférer les apprentissages.

17 Ainsi, un travail en coopération serait bénéfique lorsque les buts sont orientés vers la maitrise d'apprentissages et lorsque la tâche dépasse la capacité de la mémoire de travail individuel - sous réserve de restrictions de temps, en supposant que les membres ne possèdent pas les connaissances préalables nécessaires. Dans ces circonstances et lorsque les personnes ont une expérience préalable de travail en commun sur des tâches similaires, ils peuvent se distribuer les activités cognitives à accomplir et tirer parti de leurs capacités et de la communication inter-individuelle pour acquérir de nouvelles connaissances. Mais cet effet bénéfique de pouvoir partager la charge cognitive au sein d'un groupe peut être annulé par des coûts de communication et de coordination, c'est-à-dire la charge cognitive causée par les activités interactives entre les membres du groupe (ibid., p. 224). Une organisation d'un travail en groupe visant l'émergence de conflits socio-cognitifs, et pas seulement une production commune ou la réalisation d'une œuvre, nécessite donc des conditions sur lesquelles nous avons porté une attention forte dans la mise en œuvre méthodologique de cette recherche.

\section{L'activité des élèves}

Par le travail en groupe, les élèves vont chercher des solutions pour dépasser un problème, créer des nouvelles représentations et règles d'action dans la construction d'apprentissages (Bécu-Robinault \& Robin, 2017). En effet, dès que des individus échangent à propos d'une tâche, ils sont susceptibles, à un moment ou à un autre, d'être en désaccord (Darnon et al., 2006). Dans notre étude, nous souhaitons avoir accès à l'activité réalisée par les élèves en situation de travail en groupe, en analysant les conflits socio-cognitifs auxquels ils sont confrontés.

\subsection{L'approche interactionniste de l'activité des élèves}

L'intérêt porté sur l'activité des élèves n'est pas récent. Des recherches en sciences sociales montrent une préoccupation constante pour comprendre comment on peut faciliter l'apprentissage et la réussite scolaire à partir des approches différentes. Dans une revue des études consacrées à l'activité des élèves, Dieumegard (2011) distingue quatre orientations de recherche, articulées autour des dimensions cognitives et sociales: a) le solipsisme - le rapport des élèves à un milieu d'apprentissage sans explorer les phénomènes socio-interactionnels ; b) la cognition sociale - l'influence du contexte psychosocial et d'autrui sur les individus ; c) l'interactionnisme - réunissant les dimensions sociales et cognitives de l'activité des élèves et d) l'intégration individuelle-sociale - l'activité et la cognition d'un élève sont individuelles-sociales sans pour autant être produites collectivement à tout instant. Notre article s'inscrit 
dans la direction des recherches qui portent sur les interactions sociales entre élèves et l'intégration individuelle-sociale, qui considèrent que l'apprentissage n'est pas le reflet d'une seule construction individuelle, mais d'un engagement collectif et participatif, envers un enjeu commun défini par le problème étudié. À partir des interactions entre élèves ou groupes d'élèves, dans des activités scolaires, ces recherches soulignent également « l'importance des mécanismes dialogiques ou conversationnels dans la mise en circulation des représentations" associées aux processus d'apprentissage et raisonnement (Filliettaz \& Schubauer-Leoni, 2008, p. 7).

En sens large, le concept d'activité désigne « ce que le sujet fait au monde et ce qu'il se fait en faisant " et regroupe «l'ensemble des processus de transformation du monde (physique, mental, social, et souvent les trois à la fois) dans lesquels se trouve engagé un sujet dans ses rapports avec son environnement, et en même temps les transformations de lui-même s'opérant à cette occasion » (Barbier, 2011, p. 15). La prise en compte des dimensions sociales de l'activité trouve ses origines dans les travaux de Léontiev (1975) et Engeström (1987) qui relient la singularité de l'individu à la nature collective de l'activité, susceptible de mieux expliquer comment sont obtenus les objectifs collectifs (Venturini, 2012). À travers les confrontations et les coopérations observées dans les échanges, les chercheurs explorent les «indicateurs de transformations conceptuelles significatives d'un apprentissage » (Nonnon, 2008, p. 45). Dans ces activités en coopération, lorsque l'intelligence est « distribuée entre plusieurs individus, le groupe apparait comme un sujet collectif: c'est lui qui au cours de l'interaction observée progresse, vient à bout de la tâche, découvre certaines caractéristiques de l'objet »(idem). Le sujet collectif organise son activité, en vue de l'obtention d'un certain résultat, grâce à un ensemble de mécanismes matériels ou symboliques que Engeström (1987) appelle système d'activité. Pour fonctionner, ce système doit respecter une organisation du travail, des règles et rassembler des individus engagés dans des activités motivées par des buts². Ainsi, en situation de travail en groupe, «l'interaction ne se réduit pas à une situation de co-présence entre acteurs, mais implique nécessairement une orientation vers des enjeux partagés et distribués parmi les participants" (Filliettaz \& Schubauer-Leoni, 2008, p.12). L'approche interactionniste accentue ainsi le rôle de l'engagement mutuel des participants, à travers des formes d'actions communes. Le groupe d'élèves, en tant qu'individu collectif, participe à la construction des savoirs et un rôle essentiel revient aux interactions verbales, décodées grâce à l'analyse des dialogues, lors de l'activité des élèves (Dieumegard, 2011).

\subsection{Tâche ou activité ?}

21 En sens large, l'analyse de l'activité exige de prendre en compte tout ce que développe un sujet lors de la réalisation de la tâche : «non seulement ses actes extériorisés, mais aussi les inférences, les hypothèses qu'il fait, les décisions qu'il prend dans ce qu'il fait et ce qu'il se retient de faire ; l'activité comprend aussi la manière dont le sujet gère son temps, et également son état personnel - en termes de charge de travail, de fatigue, de stress, et aussi de plaisir pris au travail -, ainsi que ses interactions avec autrui dans la situation de travail» (Rogalski, 2013, p.4). Ce type d'analyse réclame d'abord une distinction déjà classique entre les concepts « tâche » et " activité ", débat qui remonte aux travaux initiés par les psychologues russes Leontiev et Vygotsky. Ainsi, la tâche « correspond à la consigne de travail, avec le mode de résolution canonique qu'attend 
l'expert parce qu'il le connaît » et l'activité se définit par « la signification que l'acteur est en mesure de conférer à cette tâche, à la représentation qu'il s'en fait, à la diversité des procédures qu'il met en œuvre de manière effective pour la résoudre » (Astolfi, 2008, p. 60). Cette distinction est illustrée dans la Figure 1.

Figure 2 : Distinction entre tâche et activité (Astolfi, 2008, p. 61).

Figure 2: Distinction between task and activity (Astolfi, 2008, p. 61)

\begin{tabular}{|c|c|c|c|}
\hline \multirow[b]{2}{*}{$\begin{array}{l}\text { LOGIQUE DE } \\
\text { L'EXPERT }\end{array}$} & \multicolumn{2}{|c|}{$\begin{array}{c}\text { DISPOSITIF } \\
\text { (instauré) } \\
\text { Consignes, programmation, supports }\end{array}$} & \multirow[b]{2}{*}{$\begin{array}{l}\text { LOGIQUE DU } \\
\text { NOVICE }\end{array}$} \\
\hline & $\begin{array}{c}\text { TÂCHE } \\
\text { (proposée) } \\
\text { Analyse à priori } \\
\text { Opérations logiques requises } \\
\text { par la résolution canonique } \\
\text { attendue }\end{array}$ & $\begin{array}{c}\text { ACTIVITÉ } \\
\text { (induite) } \\
\text { Analyse en situation } \\
\text { Procédures et stratégies } \\
\text { effectivement adoptées par } \\
\text { les apprenants }\end{array}$ & \\
\hline
\end{tabular}

L'organisation conçue et gérée par l'enseignant ainsi que la façon dont il met en œuvre la tâche confiée aux élèves, les difficultés qu'ils rencontrent et qu'ils surmontent sont des éléments-clés pour l'activité des élèves (Ginestié \& Tricot, 2013). Certains élèves se retrouvent face à des consignes qui ne sont pas claires : ils ne savent pas s'il s'agit de faire ou d'apprendre. Pour l'enseignant, elles sont parfois de l'ordre de l'évidence, alors qu'elles permettent diverses interprétations. L'élève interprète la consigne ainsi que le " discours de l'enseignant » (Sensevy \& Quilio, 2002). Il redéfinit la tâche en fonction de ce qu'il sait et des représentations qu'il a des savoirs en jeu (Magendie, 2009 ; Magendie \& Bouthier, 2012). En réalité, ce sont les tâches redéfinies, devenues effectives, qui permettent partiellement aux observateurs (chercheurs ou enseignants) de déchiffrer comment les élèves résolvent un problème et font face aux conflits avec lesquels ils se confrontent (Magendie, 2009). Il semble prépondérant que la situation d'enseignement ait certaines caractéristiques pour que le professeur « dévolu » à l'élève le "pouvoir d'agir " et la possibilité de construire de nouvelles connaissances et de devenir responsable de cette construction (Tiberghien \& Vince, 2015). Si l'enseignant propose des tâches que l'élève a déjà effectuées, il n'apprendra pas. S'il propose des tâches déstabilisantes, il risque de créer l'effet opposé si les élèves s'interdisent d'entrer dans les attendus intellectuels, perçus comme trop difficiles ou angoissants. L'enseignant fait en sorte de "créer des énigmes" (Meirieu, 2016) en proposant des activités qui valorisent les niveaux des élèves, tout en restant dans la zone proximale de développement (Vygotski), adaptées au défi intellectuel à relever.

Rappelons que dans une situation de travail en groupe, plusieurs dérives s'y retrouvent (décrites plus haut). Elles soulignent que l'activité des élèves attendue par l'enseignant dans les tâches collectives qu'il propose et l'activité réelle des élèves dans ces tâches peuvent différer. Certains élèves s'organisent autour de ceux qui ont déjà les savoirfaire, en se focalisant sur le résultat lui-même (dérive productiviste et parasitaire). D'autres élèves recherchent un consensus de complaisance (Hugon, 2003): ils se mettent d'accord, ce qui leur permet d'éviter les conflits (dérive fusionnelle et bavarde). Le groupe cherche spontanément la communication hors sujet focalisée sur soi-même, plutôt que celle orientée vers les objectifs de travail. Cette dérive, relative à l'absence de postures de secondarisation (Bautier \& Goigoux, 2004) ou à l'existence de curricula invisibles (Netter, 2018), voit des élèves, souvent ceux les plus éloignés de la culture scolaire, attacher plus d'importance à l'organisation de la coopération, plutôt 
qu'à ce qu'ils ou elles peuvent retirer de la situation de travail. Lorsqu'on les interroge sur ce qu'ils ou elles ont appris, la réponse est « travailler avec d'autres » et certain-e-s expliquent même s'être mis-e-s en retrait pour laisser les plus compétent-e- $s$ réussir le travail.

\subsection{Des activités en partie inaccessibles} difficile pour certains élèves, car, dans ces phases, l'enseignant n'influence pas, ni par des aides, ni par des indications, directes ou indirectes (Raab, 2014 ; Robert \& Hache, 2013). Pour construire de nouveaux savoirs dans les situations d'apprentissage, l'élève doit rencontrer et dépasser l'obstacle dans la réalisation de la tâche (Astolfi, 1992 ; Meirieu, 2016). Dans les moments d'autonomie, sans la régulation directe de l'enseignant, certains élèves ne se confrontent pas à l'obstacle didactique dans de bonnes conditions, voire ne parviennent pas à l'atteindre et sont stoppés en amont. Certaines activités ne sont pas ainsi accessibles pour tous les élèves. Celles appelées $a$ maxima sont spécifiques pour les élèves qui débutent leur travail dès que le professeur les sollicite, en autonomie et avec une capacité à surmonter les adaptations demandées. À l'inverse, les activités a minima sont typiques pour les élèves plus distraits, moins autonomes, susceptibles d'attendre le dernier moment pour travailler (Robert \& Hache, 2013). En tant que lycéens, habitués à être dans une modalité de travail par «cours dialogué » (Veyrunes \& Saury, 2009), ces élèves n'obtiennent pas de "rétroaction » rapide (Brassard, 2012), d'autant plus lorsque l'enseignant, pour les laisser travailler en groupe, s'interdit de « dire le savoir " (Brousseau, 1998). C'est pour cela que la mise en activité des élèves est conditionnée par la clause de la "réticence didactique " (Sensevy \& Quilio, 2002) et déterminée par l'enseignant qui doit «cacher » une partie de ce qu'il sait et résister au piège de l'effet Topaze ${ }^{3}$ et de la "pédagogie bavarde " (Meirieu, 2016).

S'intéresser à l'activité des élèves a comme objectif une meilleure compréhension du processus d'apprentissage de savoirs formalisés, de ses transformations, de la manière dans laquelle une connaissance est abandonnée pour laisser la place à une nouvelle, plus fonctionnelle et capable de leur permettre de résoudre des problèmes qu'ils ne pouvaient résoudre auparavant (Ginestié \& Tricot, 2013). Étudier leur activité dans des situations considérées « difficiles » permet de révéler aussi ce qu'ils peuvent faire, mais aussi ne pas faire, dans la réalisation des tâches données (Magendie, 2009). Cela permettrait aux observateurs (chercheurs, formateurs ou enseignants) de mieux déchiffrer les manières selon lesquelles les élèves d'une classe, réunis en groupe, résolvent une tâche à laquelle ils sont confrontés, les difficultés et les obstacles qu'ils doivent surmonter. Robert et Hache attirent l'attention sur l'action elle-même, qui, même répétée, «n'engendre pas nécessairement une construction de connaissances, il faut une transformation de cette action en activité » (2013, p. 47). Déterminées par des facteurs variés (notamment par le sujet lui-même), on anticipe que ses activités ne seront pas les mêmes pour tous les élèves qui ne suivront pas le parcours d'un même «itinéraire cognitif» pour les résoudre. Dans le même sens, Dieumegard avertit que pour « analyser l'activité des élèves à l'échelle d'une classe, on ne peut pas se contenter d'un ou deux élèves, et l'on devrait recueillir ces données pour chacun d'entre eux, ce qui est théoriquement possible, mais pratiquement difficile» (2011, p. 40). C'est en ce sens que nous montrons à voir, dans cet article, l'activité réalisée par tous les élèves de 
plusieurs groupes, à travers une partie de leurs comportements et de leurs locutions verbales. Toutefois, les résultats de cette étude ne pourront dévoiler que des reconstitutions de traces observées des situations, car les activités réelles (tout ce que l'élève fait et comment il fait) restent en partie inaccessibles (Robert \& Hache, 2013).

\section{Problématique et question de recherche}

Les pratiques de coopération entre élèves ont longtemps été étudiées pour aborder la dimension sociale dans l'apprentissage, autrement dit, pour apporter des éléments de réponse à la question: apprend-on mieux à plusieurs? De nombreux travaux en psychologie ont mis à jour plusieurs conceptions de l'enseignement qui recourent à des formes diversifiées de coopérations. Plus récemment, avec l'avènement des neurosciences éducatives, cette dimension a été mise de côté, principalement parce que la technologie utilisée par ces chercheurs (les IRM fonctionnnels) ne leur donne pas la possibilité d'observer des sujets en interaction. En conséquence, ces recherches ont peu investigué du côté de la dimension sociale des apprentissages : « la théorie de la charge cognitive ne s'est intéressée que sporadiquement à certains détails de l'apprentissage collaboratif » (Kirschner et al., 2018, p. 213). Mais ces recherches sur le fonctionnement de la cognition ont considérablement permis l'avancée des savoirs sur l'acte d'apprendre. "Grâce à l'avancée de l'imagerie cérébrale et des recherches en neurosciences, nous connaissons de mieux en mieux comment le cerveau fonctionne et rend possible l'apprentissage" (Masson, 2020, p. 8). Elles mettent en exergue de nombreux repères pour orienter l'acte d'enseigner vers une ingénierie pédagogique éclairée (Favre, 2015, Masson, 2020 ; Musial \& Tricot, 2020 ; Sander et al., 2018).

Cette meilleure compréhension des phénomènes cognitifs entre toutefois en tension avec la progression des recherches en matière de pédagogies de la coopération, tenant compte rarement des savoirs récemment construits en matière de cognition. Les travaux en psychologie sociale s'appuient faiblement sur ceux en psychologie cognitive (et inversement). De même, les travaux en pédagogie et en sciences de l'éducation ont beaucoup tendance à se méfier du champ de la neuroéducation, critiquée pour des penchants scientistes, biologisants et néolibéraux (Blais \& Laval, 2019; Caussidier, 2014 ; Giordan, 2015; Rousset, 2016). À ce jour, malgré de nombreux travaux sur la dimension sociale de l'apprentissage, peu de recherches en pédagogie ont étudié le lien entre cognition et coopération. Nous avons toutefois pu nous appuyer sur le point de vue de l'activité des enseignants mettant en œuvre des classes coopératives en collège (Leblanc \& Sauvaire-Maltrana, 2019) ou de l'avis des élèves inscrits dans ces classes (Connac, 2018a). Cet article ne prétend pas appréhender l'ensemble des possibles coopératifs, il fait le choix de restreindre son objet aux activités de travail en groupe d'élèves ${ }^{4}$, pour détailler cette forme scolaire au regard de l'objectif de susciter des conflits socio-cognitifs. Il s'agit d'étudier en quoi faire travailler des élèves en groupe au cours d'une séance d'enseignement peut les aider à engager un processus d'apprentissage, jusqu'à la conscientisation du besoin d'apprendre. Autrement dit, par l'intermédiaire de l'étude de l'activité d'élèves travaillant en groupe, nous allons explorer ce que la technologie des neurosciences ne permet pas encore d'observer : les interactions entre eux et l'émergence des désaccords par les échanges sociaux. activités de confrontations d'idées produites par des élèves travaillant en groupe ? Elle 
propose ainsi de témoigner de l'activité coopérative d'élèves travaillant en groupe (en classe de seconde lors d'enseignements en Physique-Chimie et Mathématiques) et d'étudier plus finement les circonstances et les conditions apparentes d'émergence de conflits socio-cognitifs de cette configuration coopérative de travail.

Nous tenterons donc de décrire l'activité constatée d'élèves en situation de travail en groupe, pour en étudier ensuite les éventuelles conséquences pédagogiques. La recherche est ici entendue comme « accompagnant les processus de transformation du monde et portant sur ces processus » (Barbier, 2011, p. 6). Il s'agit donc d'observer le rapport épistémique au savoir d'élèves pendant un travail en groupe. C'est une étude de la nature des interactions verbales entre élèves lors de travaux en groupe, dans des disciplines différentes. L'enjeu est, dans un premier temps, d'observer ce que disent des élèves lorsqu'ils sont conduits à travailler en groupe, après avoir cherché individuellement, afin d'observer les éventuelles confrontations cognitives. Dans un second temps, il s'agit d'étudier les conditions apparemment nécessaires pour susciter le développement partagé de ces litiges cognitifs entre élèves.

\section{Méthodologie}

La méthodologie employée dans cette recherche s'inscrit doublement dans une démarche collaborative et dans une analyse par «théorie ancrée " (Glaser \& Strauss, 2010). Elle s'appuie sur une méthode d'enquête qualitative orientée vers une production finale, ici à caractère pédagogique, souhaitant développer des postures critiques des théories en vigueur sur le travail en groupe d'élèves. Il ne s'agit donc pas seulement de décrire des situations d'activités d'élèves travaillant en groupe, mais aussi de leur donner un sens pour tendre ensuite vers la mise en exergue d'abstractions utiles à l'organisation de cette modalité scolaire.

31 Nous avons donc collecté des données relatives à du travail en groupe d'élèves, méthodiquement retranscrit les locutions enregistrées, puis procédé à leur analyse du point de vue de la présence ou l'absence de conflits socio-cognitifs, à travers les transactions et négociations repérées.

\subsection{L'organisation du travail en groupe}

Les enseignants associés à cette recherche collaborative se sont entendus pour introduire diverses précautions afin d'organiser le travail en groupe d'élèves :

- la tâche était centrée sur une situation-problème, caractérisée par la présence d'un obstacle intellectuel dépassable par l'intermédiaire du savoir à enseigner (De Vecchi, 2017, p. 124) ;

- un temps collectif de présentation du problème est conduit par l'enseignant, jusqu'à ce que les élèves n'aient plus de question à lui poser au sujet de sa compréhension et soient en situation de travailler adéquatement (Sensevy et al., 2020, p. 66) ;

- les groupes ont été constitués à partir d'élèves volontaires, avec une taille réduite de 4 ou 5 membres (Kirschner et al., 2018), sur la base de composition aléatoire (pour éviter les regroupements d'élèves faibles ou socialement discriminés) ;

- les élèves ont préalablement réfléchi seuls, pendant 3 minutes, au problème distribué, afin de s'approprier le problème et d'être en mesure d'apporter des idées au groupe ;

- la durée du travail en groupe a été de 5 à 10 minutes, avec un rappel initial de la consigne d'échanger sur les désaccords ; 
- des rôles ont été distribués aux élèves pour indiquer clairement qui a la responsabilité de quoi et pour, de ce fait, réduire la charge supplémentaire causée par les activités transactives (Kirschner et al., 2018, p. 228) ;

- après ce travail en groupe, un élève " porte-parole » a énoncé devant la classe les différentes idées échangées. L'enseignant les a toutes notées au tableau, avant qu'il reprenne la main sur la conduite des échanges. Mathématiques. En Physique-Chimie, les élèves ont disposé d'un temps de réflexion individuelle de $2 \mathrm{~min}$, pour écrire leurs idées sur une feuille. Ensuite, l'enseignante a créé les groupes par tri aléatoire, soit 7 groupes de 4 élèves. Après leur avoir demandé de se répartir des rôles précis (rédacteur, rapporteur, référent du temps, distributeur de parole, gestionnaire du niveau sonore) et avoir rappelé de chuchoter, la consigne du travail en groupe, pendant 5 minutes était de « confronter vos réponses, argumenter sur quoi vous n'êtes pas d'accord» (AC01, AD01). Une fois la consigne expliquée, l'enseignante a adopté une posture de retrait et est intervenue uniquement pour rappeler le temps ou faire appel à l'ordre.

lors des séances de recherche collaborative). Il a été observé que les enseignants de Mathématiques n'avaient pas prévu de répartition des rôles et donnaient davantage de temps pour travailler en groupe (10 minutes, est rappelée) : « le but n'est pas que vous soyez d'accord, le but c'est d'échanger... confronter vos idées ».

Ce terrain proposé par les enseignants a été investi pour une «utilisation circonstanciée des référents interprétatifs » (Paillé \& Mucchielli, 2012, p. 131). Il n'a donc pas fait l'objet d'une préparation spécifique pour les chercheurs, au-delà de ce qui avait été estimé nécessaire pour les élèves. Le terrain a uniquement donné à voir des élèves en activité de travail en groupe, autour d'une même situation-problème. Le protocole d'analyse de l'activité d'élèves, qui reste un engagement supplémentaire par rapport à l'activité originelle des élèves (Barbier, 2011, p. 10), aboutit à des conclusions théoriques inhérentes à l'émergence de désaccords et d'incertitude par l'organisation de travaux en groupe.

\subsection{Participants et recueil de données}

Deux classes de seconde d'un lycée public d'une ville du sud de la France regroupent une équipe d'enseignants qui souhaite développer un projet de pédagogies coopératives. Il s'agit de deux classes ordinaires, regroupant essentiellement des élèves non-volontaires, ignorant travailler avec des enseignants s'étant accordés pour développer des pédagogies coopératives. Quelques moyens ont été accordés pour organiser du co-enseignement : les élèves disposent de deux heures contiguës pendant lesquelles les enseignants organisent des ateliers. La politique de l'établissement est plutôt de ne pas constituer de classes à projet pédagogique spécifique, mais de disséminer des outils coopératifs dans l'ensemble des classes. C'est dans ce contexte que les élèves ont été confrontés à du travail en groupe.

La présente recherche a été conduite de manière collaborative avec les enseignants intervenant dans ces deux classes ${ }^{5}$. Une recherche collaborative est co-construite par un collectif associant enseignants-chercheurs et enseignants-praticiens. Pendant deux années, l'équipe collaborative s'est retrouvée régulièrement, d'abord pour échanger sur 
les pratiques coopératives et la dimension sociale des apprentissages (à partir de lectures croisées), puis pour construire le dispositif de recherche autour des séances avec du travail en groupe (selon une organisation sociocratique des échanges et des prises de décisions), enfin pour étudier les données collectées et en pratiquer un croisement d'analyses.

Ces recherches "avec" se déterminent en effet lorsque chercheurs et praticiens s'associent pour conduire une démarche de recherche en tant que co-auteurs, de sa conception à sa publication. Les objectifs de la recherche, la question centrale éprouvée, les modalités de collecte des données ainsi qu'une partie de leur analyse, la détermination des conclusions et des éléments de rédaction sont conjointement discutés, décidés, réalisés et évalués. «Le chercheur ne pose pas, par son choix d'objet, un regard normatif et extérieur sur ce que font les enseignants, mais va chercher, avec eux, et de l'intérieur du contexte dans lequel ils exercent, à comprendre ce qui supporte leur agir » (Desgagné, 1997, p. 374). À l'instar de Bruno Robbes (2013), nous pensons que les recherches « avec » représentent un principe de nature à orienter des collaborations futures entre équipes de pédagogues et équipes de chercheurs.

Les recherches « avec » diffèrent des recherches « sur » qui se traduisent par l'accueil de chercheurs par des enseignants : la recherche s'intéresse à l'activité enseignante pour développer un processus d'administration de conclusions de recherches, en vue de publications. Les enseignants ne sont pas à l'initiative de ces immersions en contexte professionnel, ce sont les chercheurs qui ont besoin de données empiriques pour tester des hypothèses ou éprouver des protocoles préalablement conçus en "laboratoires ". Les enseignants sont considérés comme des objets d'investigation. C'est sur leurs pratiques que les chercheurs posent un regard distant et évaluatif (Desgagné, Bednarz, Lebuis, Poirier, \& Couture, 2001).

Le concept de recherche collaborative prend forme autour de l'idée de faire de la recherche " avec » plutôt que "sur » les praticiens (Lieberman, 1986). Ils deviennent, à un moment ou à un autre de la recherche, les co constructeurs de la connaissance à produire liée à l'objet investigué (Cole, 1989). Des enseignants-chercheurs (3) se sont donc associés à des enseignants-praticiens (12) réunis en équipe pour définir ensemble la thématique générale de leurs recherches. Ils ont ensuite bâti le cadre conceptuel et théorique, ainsi que la problématique à éprouver. «Cette activité réflexive s'appuie essentiellement sur l'explicitation et l'analyse de situations de pratique vécues par les enseignants, sous l'angle de l'intérêt commun défini par le projet d'exploration. C'est dire que l'activité est aménagée de telle sorte qu'elle favorise et fait en sorte que soit entretenue une sorte de "conversation", pour emprunter à Schön, entre la pratique (des enseignants) et le retour réflexif sur cette pratique (entre praticiens et chercheurs). Les enseignants-chercheurs se sont chargés des contingences méthodologiques, relatives au dispositif de collecte de données et d'analyse du matériau collecté. Concrètement, l'activité prend forme à travers des rencontres régulières entre chercheurs et praticiens, rencontres qui permettent ainsi de créer une "zone interprétative" autour de la pratique qui est objet d'exploration " (Desgagné et al., 2001, p. 38). C'est ainsi que le choix du travail en groupe, spécifiquement l'étude de l'émergence potentielle de conflits socio-cognitifs, a été effectué. C'est pour cela que cette recherche collaborative n'a pas pris la forme d'une recherche-intervention (Marcel, 2015) puisque les chercheurs ne sont pas intervenus pour répondre à une 
demande des enseignants, mais pour conduire avec eux une étude relative à leur contexte professionnel.

41 Ces situations de recherche-collaborative ont été accompagnées par des comptesrendus rédigés à plusieurs ${ }^{6}$ et systématiquement envoyés à tous les membres de l'équipe, afin que les absences de certains ne les conduisent pas à se sentir dépassés par l'avancée des travaux et que le collectif puisse faire progresser le projet de recherche. Ces réunions de recherche collaboratives peuvent de la sorte constituer une occasion de formation continue pour les enseignants à qui l'on propose une analyse de leurs pratiques en vue de les éclairer et de les améliorer.

Joëlle Zask (2011) présente trois critères qui orientent les recherches collaboratives, que l'on a retrouvé à travers cette expérience : elles sont d'abord une occasion pour les non-chercheurs de prendre part à une recherche et donc de participer, par le truchement des méthodologies employées, à la consolidation de leurs compétences en matière de posture réflexive et d'auto-évaluation professionnelle. Elles sont ensuite le lieu de leur écoute, de leur participation à l'enrichissement des données. Elles visent enfin des bénéfices pour les enseignants, principalement dans leur champ d'action. Il s'agit plus d'augmenter leur puissance d'agir en tant qu'enseignant que de leur faire miroiter une éventuelle orientation professionnelle.

43 La démarche de la recherche menée avec cette équipe de professeurs peut être résumée ainsi :

Étape 1. Présentation du projet aux enseignants volontaires ;

Étape 2. Travail sur les représentations des collaborateurs - dans le champ de la recherche conduite - échanges sur les lectures de recherches sur la coopération entre élèves et la dimension sociale des apprentissages ;

Étape 3. Détermination de la problématique et des hypothèses à tester Détermination du dispositif pédagogique à étudier ;

Étape 4. Organisation de la collecte de données, à partir du cadrage méthodologique défini par les enseignants-chercheurs : qui fait quoi? Comment? Avec quelles échéances? Quelles dates? Quels regroupements? Quelles modalités de communication?

Étape 5. Organisation du traitement des données : qui fait quoi ? Comment?

Étape 6. Croisement des analyses - Distanciation et théorisation - Écriture des conclusions de la recherche ;

Étape 7. Rédaction de l'article par les enseignants-chercheurs.

Les participants à cette recherche sont des enseignants et des élèves d'un lycée de Béziers, observés pendant des activités de travail en groupe, menées en cours de Mathématiques et de Physique-Chimie. Le recueil de données a été effectué en enregistrant en audio et en vidéo les interactions observables, verbales et non verbales des élèves dans les situations de travail en groupe. L'enregistrement audio et vidéo a été fait en assurant les participants de respect de l'anonymat et l'utilisation des données à but exclusivement scientifique. Dans certains groupes la présence de la caméra a été un facteur de distraction.

Les enseignants-chercheurs se sont entendus avec les enseignants-praticiens pour pouvoir procéder à une collecte de données pendant la réalisation de travaux en groupe lors de certains de leurs cours. Sur l'ensemble du collectif, seuls trois professeurs (une en Physique-Chimie et deux en Mathématiques) ont pu accueillir des enseignants-chercheurs. Après une période d'immersion nécessaire à l'acculturation mutuelle des élèves et des chercheurs, les données ont été saisies par l'intermédiaire d'outils technologiques précis : des enregistreurs MP3 pour la voix des élèves ainsi que 
des caméras Go-pro pour les images. L'accord des élèves a été doublement sollicité : via une autorisation de droits à l'image et suite à la demande individuelle d'enregistrement juste avant d'installer le matériel. Ainsi, pour au moins deux groupes par cours, chaque ilot a été équipé d'un enregistreur MP3 (déposé au centre de la table) et d'une caméra placée à distance en plan fixe afin de capter l'activité de tous les élèves du groupe. Par l'intermédiaire du logiciel de montage vidéo Camtasia Studio, nous avons ensuite pu synchroniser l'image et le son afin de disposer d'une vidéo par séance de recherche.

À partir des vidéos transcrites, nous les avons complétées avec les documents mis à disposition par les enseignants (préparation de cours, consigne, énoncés des exercices) ainsi que leurs retours verbaux lors des séances liées à la recherche collaborative (consistant à échanger autour des vidéos prises en classe). Nous avons ensuite effectué les analyses qui nous permettent d'associer les renseignements et de proposer une lecture des verbatims et donc des activités possibles des élèves dans une séance donnée. Pour cela, à partir des transcriptions et des vidéos, chaque séance a été découpée en épisodes spécifiques pour les moments de travail en groupe (environ 5-10 minutes). Ainsi, dans ces épisodes, nous avons retenu la situation-problème étudiée, la consigne, la durée du travail des élèves sur la tâche proposée et les échanges susceptibles de provoquer des conflits socio-cognitifs. Le tableau de verbatim a été structuré selon plusieurs colonnes: la localisation temporelle, le codage de l'occurrence, la retranscription des propos, une colonne par élève ( $F$ pour fille et $G$ pour garçon). Ont été grisées les cases indiquant la prise de parole de l'élève. Des indicateurs non-verbaux ont également été donnés dans ces colonnes.

En appui sur tous ces éléments, notamment le suivi individuel des élèves, des analyses de contenu (Bardin, 1997) de verbatims ont été réalisées en situant l'activité de chaque élève au regard de celles de ses partenaires de groupe. Les textes d'analyses permettent ainsi de décrire le fil des échanges, d'identifier les situations de désaccords entre élèves (lorsqu'elles se produisaient) et de proposer une typologie des activités réalisées par les élèves en groupe devant la tâche donnée, dans la veine des théories ancrées décrites précédemment. Ces moments permettent également de mettre en évidence, en partie, l'autonomie laissée aux élèves et la manière avec laquelle ils dépassent les possibles obstacles identifiés lors de leur activité.

Dix-sept situations de travail en groupe ont ainsi été filmées. Toutes ont été retranscrites littéralement puis analysées : cinq groupes en Physique-Chimie (de AA à $\mathrm{AE}$ ) et douze groupes en Mathématiques (de $\mathrm{AF}$ à $\mathrm{AQ}$ ). La sélection des extraits présentés dans cet article s'est opérée selon trois critères: la possibilité de réaliser fidèlement les verbatims (à partir de la qualité d'accès complète aux voix), l'authenticité apparente des élèves devant la caméra utilisée pour enregistrer leur activité (afin d'éviter aussi souvent que possible l'effet Hawthorne ${ }^{7}$ ), l'originalité des situations présentées (des configurations similaires apparaissant dans des épisodes différents).

\section{Analyse des données}

Nous proposons ci-dessous plusieurs extraits de la transcription de dialogues (les verbatims) entre les élèves de plusieurs groupes, lors des échanges-clés pendant la réalisation de la tâche. L'analyse de ces données conduit à la mise en évidence des types 
différents d'organisation du travail en groupe, à partir desquels il est ensuite possible d'extraire des vigilances pédagogiques.

\subsection{Les groupes de Physique-Chimie}

50 Les élèves ont été placés devant une situation-problème : «Voilà une pierre suspendue à un fil élastique, elle est immobile. Quels sont les objets qui agissent sur la pierre ? » La notion didactique attachée à cette situation-problème est celle d'action mécanique : l'effet d'un objet A qui agit sur un objet B. Cette action est modélisée par une force, représentée comme un vecteur (flèche) dont il faut préciser l'origine (point de départ), la direction (droite), le sens (vers où pointe la flèche) et l'intensité (la longueur de la flèche). Dans cette situation d'une pierre immobile sous un élastique, il n'y a que deux objets qui agissent sur la pierre : l'élastique et la Terre.

51 Voici le verbatim de ce qui concerne directement les conflits d'idées :

Extrait $1:$ Verbatim du groupe 1.

Extract 1: Group 1 verbatim

\begin{tabular}{|c|c|c|c|c|c|c|}
\hline & $\mathrm{AC}$ & Verbatim & F1 & F2 & F3 & G1 \\
\hline $22^{\prime 22}$ & $\mathrm{AC} 06$ & $\begin{array}{l}\text { Pourquoi barre de fer ça me fait rire ? + La barre de } \\
\text { fer ça fait partie du support }\end{array}$ & & & & \\
\hline 2,27 & $\mathrm{AC} 07$ & T'es sûr? & & & & \\
\hline $2 ' 28$ & $\mathrm{AC} 08$ & Certain + Léa t’as mis quoi? & & & & Sollicite F1 \\
\hline 2,30 & AC09 & J'ai mis pareil, j'ai rajouté la gravité & & & & \\
\hline 2,33 & $\mathrm{ACl} 0$ & Moi j'ai mis l'élastique & & & & S'adresse à F1 \\
\hline $2 ' 35$ & $\mathrm{ACl1}$ & Mais ce n'est pas un objet & & $\begin{array}{l}\text { S’adresse à } \\
\text { F1 }\end{array}$ & & \\
\hline 2,36 & $\mathrm{ACl2}$ & $\begin{array}{l}\text { La Terre est un objet et c'est la Terre qui produit la } \\
\text { gravité }\end{array}$ & & & & Répond à F2 \\
\hline 2,37 & $\mathrm{ACl3}$ & La question c'est quels sont les objets & & & & \\
\hline $2 ' 39$ & $\mathrm{ACl} 4$ & La Terre la Terre la Terre est un objet & & $\begin{array}{l}\text { N'est pas } \\
\text { d'accord }\end{array}$ & & $\begin{array}{l}\text { Insiste auprès } \\
\text { de F2 }\end{array}$ \\
\hline $2 ' 44$ & $\mathrm{AC} 15$ & La Terre c'est pas un objet réel & & & & \\
\hline $2 ' 46$ & $\mathrm{ACl6}$ & $\begin{array}{l}\text { Alors c'est que l'élastique parce que la pierre elle } \\
\text { touche pas le support on est d'accord? }\end{array}$ & & & & \\
\hline $2 ' 50$ & $\mathrm{ACl} 7$ & Oui mais elle est suspendue au support & $\begin{array}{l}\text { N'arrive pas } \\
\text { à se faire } \\
\text { entendre }\end{array}$ & & & \\
\hline 2,52 & $\mathrm{ACl} 8$ & Oui mais elle est sur le support & & & $\begin{array}{l}\text { S'adresse } \\
\text { à Gl }\end{array}$ & \\
\hline 2,54 & $\mathrm{ACl} 9$ & Oui l'élastique est sur le support pas la pierre & & & & Répond à F3 \\
\hline 2,56 & AC20 & Non mais l'élastique il tient la pierre & & & $\begin{array}{l}\text { Répond à } \\
\text { G1 }\end{array}$ & \\
\hline 259 & $\mathrm{AC} 21$ & $\begin{array}{l}\text { L'élastique tient la pierre et l'élastique est tenu par le } \\
\text { support }\end{array}$ & Synthétise & & & \\
\hline $3{ }^{\prime} 01$ & $\mathrm{AC} 22$ & $\begin{array}{l}\text { Donc c'est l'élastique qui agit sur la pierre pas le } \\
\text { support }\end{array}$ & & & & Répond à F1 \\
\hline $3{ }^{\prime} 03$ & $\mathrm{AC} 23$ & Le support agit sur l'élastique qui agit sur la pierre & & & & \\
\hline $3 \cdot 05$ & $\mathrm{AC} 24$ & Donc, ça agit & & & $\begin{array}{c}\text { S’adresse } \\
\text { à Gl }\end{array}$ & \\
\hline $3 ’ 06$ & $\mathrm{AC} 25$ & Mais ya aussi la gravité & $\begin{array}{l}\text { Parle au } \\
\text { groupe }\end{array}$ & & & \\
\hline 3,08 & $\mathrm{AC} 26$ & $\begin{array}{l}\text { Ouais déjà moi je pense il faut mettre la Terre sinon } \\
\text { le truc il serait là-haut }\end{array}$ & & & & $\begin{array}{l}\text { Montre le ciel } \\
\text { du doigt }\end{array}$ \\
\hline $3 ' 16$ & AC27 & Ce serait comme dans l'espace & Répond à Gl & & & \\
\hline $3 ' 17$ & AC28 & Du coup, on fait quoi? & & & $\begin{array}{l}\text { S'adresse } \\
\text { à G1 }\end{array}$ & \\
\hline $3 \cdot 19$ & $\mathrm{AC} 29$ & Moi je sais pas & & & & \\
\hline
\end{tabular}

Dans ce groupe, un conflit socio-cognitif se produit au sujet de la place de la Terre ou de la gravité dans les forces qui jouent sur la pierre : «t'es sûr? " (AC07). Une élève se demande si ce qui tient la pierre fait partie, ou pas, du support. Un de ses partenaires l'affirme. Elle le lui demande s'il en est certain et il passe à autre chose. Son visage manifeste des signes de doute. Un second se produit au sujet de l'intervention du support ou de l'élastique (AC21) : les élèves se demandent si la pierre est tenue par le support ou par l'élastique qui la relie au support et interrogent l'impact de la gravité. La situation se termine par l'expression d'une incertitude : « Moi je sais pas » (AC 3'19). 
53 Les quatre élèves de ce groupe semblent s'être approprié la situation-problème. Tous participent à sa résolution. Plusieurs conflits socio-cognitifs apparaissent sous la forme de désaccords ou de doutes entre élèves. L'ensemble de ces désaccords se produit sur 1 minute, ce qui fait de ces élèves des « coopérateurs express ». Le reste du temps est consacré à la distribution des fonctions et à des sujets-parasites. Cette distribution n'ayant pas été faite au début, les élèves terminent le travail en groupe sans revenir sur leur incertitude.

Extrait 2 : Verbatim du groupe 2.

Extract 2: Group 2 verbatim

\begin{tabular}{|c|c|c|c|c|c|c|}
\hline & AD & Verbatim & F1 & F2 & G1 & G2 \\
\hline $2^{\prime} 21$ & AD25 & L'air + puis la Terre & & & & $\grave{A}$ G1 \\
\hline $2^{\prime} 30$ & AD26 & Et toi t'es d'accord? & À F2 & & & \\
\hline $2^{\prime} 32$ & AD27 & La table & & Au groupe & & \\
\hline $2^{\prime} 36$ & AD28 & $\begin{array}{l}\text { On peut la bouger la table }+ \text { ça fait bouger } \\
\text { la pierre }+ \text { mais elle bouge trop la pierre } \\
\text { aussi }+ \text { immobile }\end{array}$ & & & & $\begin{array}{l}\text { Bouge la table } \\
\text { puis stabilise } \\
\text { la pierre }\end{array}$ \\
\hline $2{ }^{\prime} 56$ & AD29 & Regarde ce qu'ils font eux ? & À G2 & & & $\begin{array}{l}\text { Regarde } \\
\text { derrière lui }\end{array}$ \\
\hline $3^{\prime} 04$ & AD30 & Du coup la table aussi ? & Au groupe & & $\begin{array}{l}\text { Ecrit sur } \\
\text { une } \\
\text { feuille }\end{array}$ & \\
\hline $33^{\prime} 06$ & AD31 & $\begin{array}{l}\text { Je sais pas tout le monde est d'accord pour } \\
\text { qu'on mette la table? }\end{array}$ & \multirow{4}{*}{ Note «la table» } & & & Au groupe \\
\hline $3^{\prime} 08$ & AD32 & Ouais vas-y & & Au groupe & & \\
\hline $3^{\prime} 17$ & AD33 & Y a quoi d'autre? & & Souffle & & Au groupe \\
\hline $3^{\prime 2} 21$ & AD34 & Y a que ça je crois & & $\begin{array}{l}\text { Répond à } \\
\text { G2 }\end{array}$ & & $\begin{array}{l}\text { Regarde } \\
\text { ailleurs puis } \\
\text { consulte son } \\
\text { téléphone }\end{array}$ \\
\hline $3^{\prime} 53$ & AD35 & $\begin{array}{l}\text { Tu l'as fait le commentaire composé ? + Tu } \\
\text { l'as fait toi ? }\end{array}$ & $\begin{array}{l}\text { Fait oui de la } \\
\text { tête }\end{array}$ & $\begin{array}{l}\text { Fait oui de } \\
\text { la tête }\end{array}$ & À G2 & $\begin{array}{l}\text { Fait oui de la } \\
\text { tête }\end{array}$ \\
\hline
\end{tabular}

54 idées puisqu'ils se disent tous d'accord (voir extrait $n^{\circ} 2$ ). De leur point de vue, cela leur permettrait de " passer en premier » (AD10). Un seul conflit socio-cognitif se produit au sujet de la place de la table comme force sur la pierre (AD27). F2 formule cette idée, G2 la teste en faisant bouger la table, pour constater que la pierre bouge : «On peut bouger la table + ça fait bouger la pierre + mais elle bouge trop la pierre aussi + immobile » AD28). Mais rapidement, un consensus est établi, personne n'émettant d'avis contraire : «Je sais pas tout le monde est d'accord pour qu'on mette la table ? Ouais vas-y » (AD32). Les échanges se tournent ensuite vers de nouvelles idées. Devant le silence des élèves de ce groupe, ils en viennent à échanger sur un sujet horsproblème, concernant un devoir à rendre dans un autre cours (un commentaire composé - AD35). La suite du travail en groupe n'aborde plus la question des forces sur la pierre. Les échanges sur le problème n'ont duré qu'une minute trente.

On observe dans ce groupe que les quatre élèves participent de manières différentes à la résolution de cette situation-problème. Une élève se met en retrait et ne réalise que sa fonction de rédactrice. Un autre élève ne fournit qu'une seule idée, puis fait autre chose. Plusieurs idées sont fournies, mais jamais débattues. Dès le départ, ces élèves se disaient en accord et rien ne s'est produit pour que des oppositions se réalisent. Un seul conflit socio-cognitif est abordé, mais ne donne pas lieu à des confrontations d'arguments. Cela induit une rapidité des échanges et un glissement vers des sujets annexes. À la fin de ce travail en groupe, le degré d'incertitude parait faible pour deux d'entre eux et léger pour les deux autres tant il n'y a pas eu de conflit. En restant chacun dans des activités séparées, les élèves de ce groupe apparaissent comme des «stratèges de l'évitement ». 


\subsection{Les groupes de Mathématiques} lançant 3 dés et en additionnant on peut obtenir plus souvent un 10 qu'un 9? Pourquoi ? » La notion mathématique attachée à cette situation-problème est la théorie des probabilités (Galilée) et expliquée grâce au paradoxe du Grand-Duc de Toscane. Celui-ci avait observé lors des parties de jeu que la somme 10 était obtenue légèrement plus souvent que la somme 9. Ce paradoxe exposé par le Grand-Duc réside dans le fait qu'il y a autant de façons de décrire 10 que 9 comme sommes de trois entiers compris entre 1 et 6 . Mais les choses sont présentées de façon trompeuse, il y a 6 façons de faire 9 ou 10, mais une probabilité est un rapport et on ne peut comparer deux quantités que si elles sont sur le même rapport. Les possibilités dénombrées par le Grand-Duc ne sont pas équiprobables : certaines sommes ont plus de chance d'être obtenues que d'autres. À partir de ce constat, Galilée construit une table qui indique de combien de manières différentes un résultat est obtenu. Il calcule que 9 peut apparaître de 25 manières différentes, alors que 10 apparaît de 27 autres manières, parmi les 216 combinaisons possibles. Il est donc plus facile d'obtenir 10 que 9, mais la différence n'est que d'1/108.

Voici le verbatim de ce qui concerne directement les conflits d'idées pour les groupes de mathématiques:

Extrait 3 : Verbatim du groupe 3.

Extract 3: Group 3 verbatim

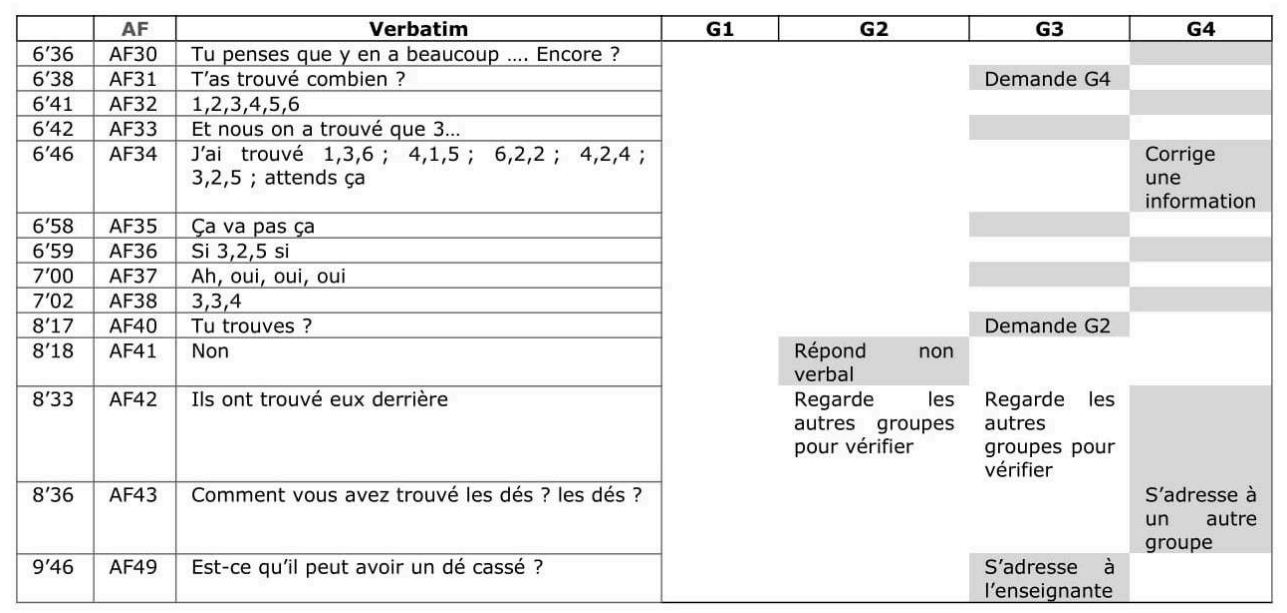

58 Au sein de ce groupe, les élèves s'engagent peu dans la tâche. G1 est soit inactif, soit il échange avec d'autres groupes ou se déplace en salle. Les élèves ne respectent pas la phase de travail individuel: ils échangent directement (voir extrait $\mathrm{n}^{\circ} 3$ ). Les discussions convergent autour de G4 qui s'occupe spontanément de la répartition des tâches (AF21) et prend l'initiative des discussions le plus souvent (AF10-AF14, AF19AF21, AF30) : «Tu penses que y en a beaucoup... Encore? » (AF30). Ses partenaires sont peu impliqués et semblent attendre sa réponse ou celle de l'enseignante.

59 Au début du travail en groupe, les élèves échangent plutôt sur la difficulté de la tâche à réaliser : «Attends, il jette combien de dès ?» (AF06-AF11). Ce moment dure près de deux minutes et les élèves montrent des signes d'incompréhension: «oui c'est compliqué » (AF12-AF21). L'intervention de l'enseignante, qui complète la consigne en 
ajoutant l'utilisation possible d'une calculatrice (AF05), semble une piste de réflexion qui est vite abandonnée: "Non, j'essaye de trouver, mais je sais plus comment s'appelle " (AF24-AF29). Les élèves sont impliqués dans la réalisation de la tâche et plusieurs pistes sont travaillées, en parallèle, sans être réellement développées. Un conflit socio-cognitif apparaît au moment où les premiers résultats des calculs à la main sont confrontés, d'abord en binôme, et ensuite en groupe (AF30-AF38). La confrontation des résultats, jugée peu satisfaisante, installe vite l'incertitude et la déception des élèves face aux autres groupes qui semblent avoir fini avant eux : «Ils ont trouvé eux derrière » (AF42) «Comment vous avez trouvé les dés? ? (AF43). Cet état de manque de confiance en soi, lié à leur capacité à résoudre l'exercice, amène les élèves dans des discussions hors sujet : «Y a eu tirage, tout à l'heure, non?» (AF45-AF48). Continuées jusqu'à l'épuisement du temps, ces discussions sont interrompues par une dernière hypothèse de travail proposée par G3 qui reste pourtant inexploitée à cause du chronomètre : «Est-ce qu'il peut avoir un dé cassé ?» (AF49). Les élèves de ce groupe peuvent être assimilés à des "complexés ", qui dévalorisent leur travail au regard de celui d'autres groupes.

Extrait 4 : Verbatim du groupe 4.

Extract 4: Group 4 verbatim

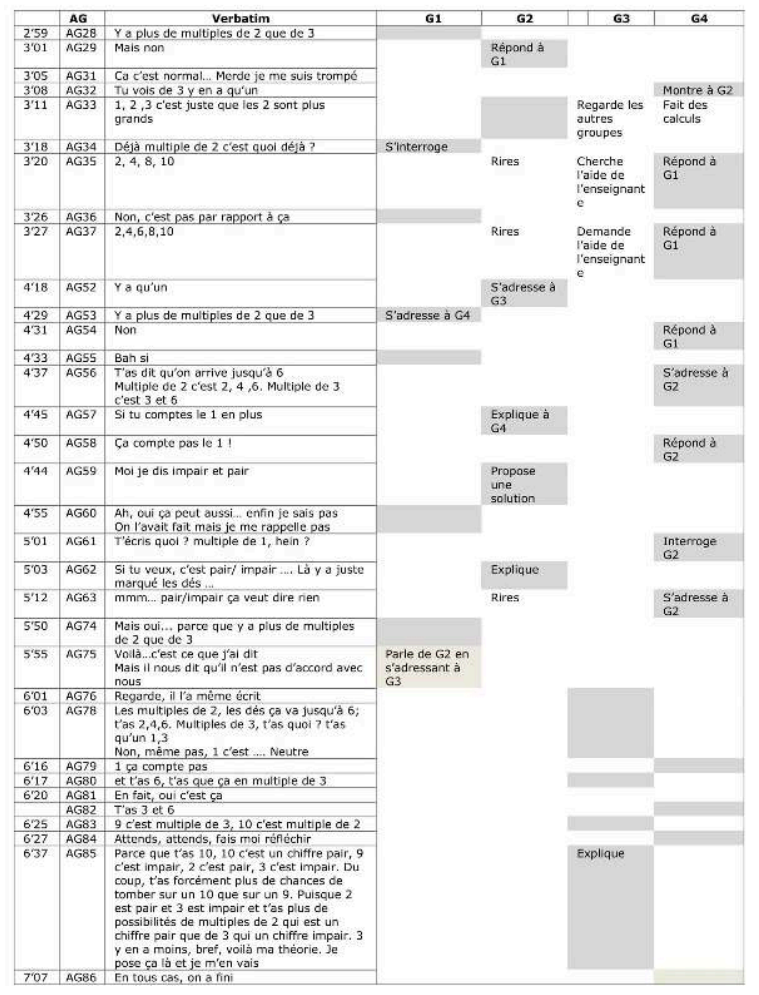

Dans ce groupe, la parole est partagée et tous les élèves participent aux échanges (voir extrait $n^{\circ} 4$ ). G1 prend spontanément le rôle de maître du temps (AG43, AG87) et initie le partage: "Alors, du coup, vous en pensez quoi? Vous en pensez quoi de cette théorie? » (AG12-AG16). Les premières discussions sont focalisées, au milieu des rires, sur la lecture et l'interprétation de la consigne qui semble peu claire pour tous: «Madame, madame, svp!» (AG12-AG23). Des doutes concernant «les multiples » et « la probabilité » ressortent plusieurs fois pendant le travail en groupe, signe que les éléments de la consigne ne sont pas suffisamment bien compris (AG40-AG52). Un 
premier conflit socio-cognitif apparaît autour des multiples. G1 et G4 estiment que sur un dé on a plus de multiples de 2 que de 3, contrairement à G2 qui les contredit en expliquant qu'il s'agit d'un nombre égal (AG53-AG63). La solution de calculer «toutes les possibilités de tomber sur 9 et sur 10 », proposée par G2 (AG64) est vite abandonnée, car c'est trop pénible, d'identifier "toutes les possibilités possibles » (AG66-AG68). Les discussions qui suivent se portent entre G1, G3 et G4, convaincus de leur raisonnement correct face à G2 qui semble se censurer au fur et à mesure devant les idées majoritaires, mais erronées de ses partenaires : «Ya pas de règle, tu tombes sur le 9 ou sur le $10 »$ (AG72). Un dernier conflit socio-cognitif vise de nouveau les discussions autour des multiples de 2 ou de $3: G 1$ s'adresse à $G 3$ pour rappeler que G2 qui « dit qu'il n'est pas d'accord avec nous » (AG75). Mais ces autres élèves ne tiennent pas compte de cette idée (AG75-AG85).

61 À la fin de cette situation, G1, G3 et G4 semblent peu dans l'incertitude et paraissent restés dans un état de certitude. Persuadés de la validité de leur réponse première « en tout cas, on a fini " (AG86), ils continuent dans des discussions hors sujet qui durent plus de 3 minutes : «Là j'ai pas mangé, mais je mange jamais, là j’ai pas mangé, mais genre, des fois je vais en face » (AG88-AG115). G2, pourtant convaincu au départ par sa solution, ne reprend plus la parole après 5 '03, laissant seulement ses partenaires s'exprimer. Cet élève s'est donc trouvé bloqué face à ces partenaires "majoritaires » qui, forts de leur statut, n'ont pas envisagé les solutions qu'il apportait au groupe.

Extrait 5 : Verbatim du groupe 5.

Extract 5: Group 5 verbatim

\begin{tabular}{|c|c|c|c|c|c|c|}
\hline & AH & Verbatim & G1 & \multirow{5}{*}{$\begin{array}{l}\text { Rires } \\
\text { Rires }\end{array}$} & \multirow{2}{*}{\begin{tabular}{|l|l|} 
G3 \\
Rires
\end{tabular}} & \multirow{2}{*}{$\begin{array}{c}\text { G4 } \\
\text { Interroge } \mathrm{Gl}\end{array}$} \\
\hline 1,38 & $\mathrm{AH} 03$ & $\begin{array}{l}\text { Ok, on va faire un tour de table pour les réponses. } \\
\text { Clément? }\end{array}$ & & & & \\
\hline 1.39 & AH04 & Non & & & Rires & \\
\hline $1 \% 41$ & AH05 & Il faut une justification. & & & Rires & Relance G1 \\
\hline $1^{\prime} 42$ & AH06 & $\begin{array}{l}\text { Bah, quand tu lances le premier dé, t'arrives jusqu'à } \\
6 \text { maximum...(inaudible) tu as moins de chance } \\
\text { après de tomber sur } d \text { autres } 6\end{array}$ & $\begin{array}{l}\text { Développe sa } \\
\text { réponse }\end{array}$ & & & \\
\hline 3,38 & AH24 & Pour moi, il faut faire un tableau de probabilités. & & & & \\
\hline 3,40 & AH25 & Eh bah, fais-le ! & & & & \\
\hline $3 \% 41$ & AH26 & J'ai oublié comme on fait (...) & & Rires & & \\
\hline $7 \times 12$ & AH65 & De la proba... & & & & \\
\hline $7 \times 16$ & AH66 & Ah, oui tu fais une base de deux comme on a traité & & & & \\
\hline 720 & AH67 & Non, non, non & Rires & & & \\
\hline $7 \cdot 23$ & AH68 & Si tu tires 1 , tu tires $1 ;$ c'est une possibilité & & & & \\
\hline $7 \cdot 27$ & AH69 & Oui & & & & \\
\hline 728 & AH70 & Ah, j'ai compris & & & & \\
\hline $7 \cdot 30$ & AH71 & C'est ultra long, mecs! & & & & \\
\hline 731 & AH72 & Oui, je sais & & & & \\
\hline 732 & AH73 & Vas- $y$, fais le! & & & & \\
\hline $7 \times 40$ & AH74 & J'en ai marre ! & & & & \\
\hline $7 \times 41$ & $\mathrm{AH} 75$ & Alors? & & & & \\
\hline $7^{\prime} 42$ & AH76 & Fais le toi, vas- $y$ ! & & & & \\
\hline $7 \cdot 44$ & AH77 & Tu songes à la réussite & & & & \\
\hline $7 \times 47$ & AH78 & Inaudible & & & & \\
\hline $7 \times 48$ & AH79 & C'est beaucoup trop long à faire & & & & \\
\hline 7550 & AH80 & Bah, oui, c'est pour ça & & & & \\
\hline 7551 & AH81 & $\begin{array}{l}\text { Il doit avoir une autre manière. Il faut que je } \\
\text { réfléchisse }\end{array}$ & & & & \\
\hline 8.36 & AH88 & Avec ça, on va trouver mais c'est long & & & & \\
\hline $8^{\prime} 40$ & AH89 & De toute façon, y a que des 9, y a pas de 10 & & & & \\
\hline & AH90 & Oui mais là t es sur le numéro 1 & & & & \\
\hline $8^{\prime} 48$ & $\mathrm{AH} 9 \mathrm{I}$ & $\begin{array}{l}\text { Attends, je crois que j'ai quelque chose } \\
\text { Je sais }\end{array}$ & & & & \\
\hline $8^{\prime} 58$ & AH92 & Vas-y & & & & \\
\hline $9 \cdot 01$ & AH93 & $\begin{array}{l}\text { Essaye avec } 2 \text { à la place de } 1 \ldots \text { et vu que c'est un } \\
\text { nombre pair, ça se terminera toujours par } 10\end{array}$ & & & & \\
\hline $9 \cdot 08$ & AH94 & Fais-le! & & & & \\
\hline $9 \cdot 12$ & AH95 & $\begin{array}{l}\text { 2, ça marche pas, j'abandonne, j'aurai pas le bac } \\
\text { Ssst }\end{array}$ & & & & $\begin{array}{l}\text { S'adresse à un } \\
\text { autre groupe }\end{array}$ \\
\hline $9 \cdot 42$ & AH98 & Il est dur & & & & \\
\hline $9 \cdot 50$ & AH99 & $\begin{array}{l}\text { Et du coup on n'a pas des explications à donner... } \\
\text { simplement parce que nous n'avons pas le niveau }\end{array}$ & & & & \\
\hline
\end{tabular}

62 Tous les élèves du groupe participent aux échanges. G4 prend spontanément l'initiative de gérer la parole tout au long de l'exercice « Ok, on va faire un tour de table pour les réponses. Clément?»(AH03), mais donne un côté théâtral aux échanges «Et toi M. Martinez qui est à ma droite? que pensez-vous ? Je vois que vous marquez pas mal 
de notes" (AH13-AH22), ce qui provoque constamment des fous rires. Pendant la discussion, les élèves semblent être plutôt en accord et peu investis dans la tâche, sans faire des efforts réels pour développer les idées proposées. Par exemple, la solution proposée par G2 de «faire un tableau de probabilités » (AH24-AH25) est acceptée par ses collègues, mais vite abandonnée (AH26). Ce moment court est suivi par des discussions hors sujet : «Il joue où, Eden Hasard?» (AH29-AH62). Quelques minutes plus tard, G2 reprend son idée de calculer les probabilités (AH65-AH66), mais encore une fois aucun élève ne souhaite se charger de cette tâche "C'est ultra long, mecs!" "J'en ai marre » (AH71-AH76) considérée « trop longue à faire » (AH79). D'autres pistes sont envisagées à partir de multiples de 2 et de 3 , mais ne semblent pas appropriées par rapport au temps restant : " Avec ça, on va trouver, mais c'est long " (AH81-AH95).

À la fin du temps de travail, les élèves semblent être dans une sorte d'incertitude, liée à la situation-problème, combinée avec un sentiment d'infériorité (AH98-AH100), exprimé plus ou moins au sérieux par les élèves « Et du coup on n'a pas des explications à donner... simplement parce que nous n'avons pas le niveau » (AH114). Ils se montrent « découragés » face à une tâche qu'ils ne parviennent pas à réaliser.

Extrait 6 : Verbatim du groupe 6 .

Extract 6: Group 6 verbatim

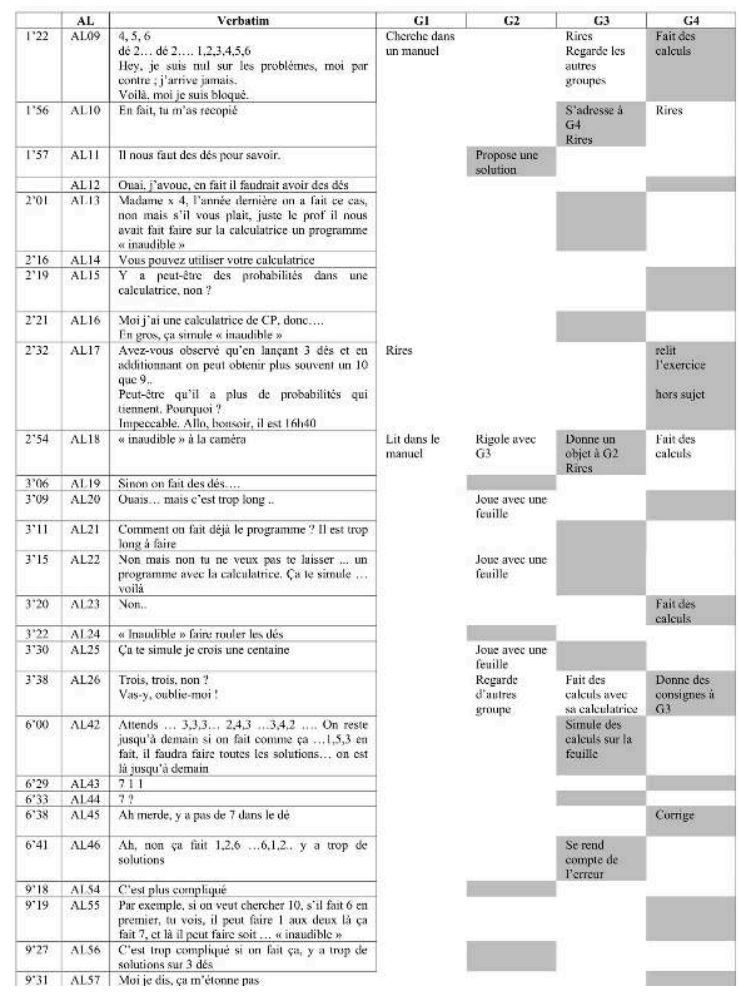

64 La répartition de la parole est assez déséquilibrée. G4 prend spontanément l'initiative des réflexions «On essaye de faire la probabilité, ok? » (AL02), accompagné ensuite de G2 (AL29) pendant que G1 (AL35) et G3 (AL25-AL32) interviennent brièvement « Faut faire que 3 lancés ». G4 a également le rôle d'organisateur du groupe quand ses partenaires ne sont plus connectés à la tâche «S'il veut parler, il parle » (AL26-AL28). Seulement deux élèves s'engagent, les deux autres sont soit inactifs, soit échangent avec d'autres groupes (voir extrait $n^{\circ} 6$ ). Pendant la situation, personne ne se préoccupe des fonctions, car cette consigne n'a été exprimée qu'à la fin : «je l'avais pas 
dit avant, mais est-ce que quelqu'un de chaque groupe peut me dire rapidement ce qu'il s'est passé dans son groupe » (AL58).

Les échanges entre les élèves évoquent plutôt des accords entre leurs points de vue. G3 vient avec la proposition de faire des dés (AL11-AL12), idée vite éliminée à cause de manque de temps « Ouais... mais c'est trop long (AL20). On observe dans ce groupe, le besoin des élèves de faire appel à la rétroaction de l'enseignante pour valider leurs hypothèses « Madame Madame Madame Madame, l'année dernière on a fait ce cas, non, mais s'il vous plait, juste le prof il nous avait fait faire sur la calculatrice un programme " (AL13). La consigne supplémentaire, donnée par l'enseignante "vous pouvez utiliser votre calculatrice » (AL14), crée des nouveaux échanges sur l'utilité d'un calculateur dans «la simulation des probabilités " (AL15; AL21-AL25). Les élèves sont engagés dans la réalisation de l'exercice pendant tout le temps qu'ils ont à disposition et semblent avoir des idées plutôt complémentaires.

Un conflit ressort au moment des discussions sur les combinations de lancées de dés. G3 affirme avoir fait 5 (AL31), pendant que G1 dit qu'il faut faire juste 3 (AL35). À partir des calculs de probabilités, $\mathrm{G} 4$ propose une solution « peut-être parce que dès qu'on tombe sur un 6 dans un des dés » (AL41), peu soutenue par G3 qui le corrige (AL44) et G2 qui considère que c'est une proposition trop compliquée à cause du nombre important de solutions (AL54-AL56).

67 Ces élèves semblent plutôt utiliser des stratégies d'évitements des conflits et de la confrontation. Les désaccords entre élèves ne concernent pas tous l'obstacle proposé par le problème. À la fin du temps de travail, pour ce groupe, deux élèves ne semblent pas en incertitude, car ils ne sont pas entrés dans la réalisation de la tâche et ont laissé leurs deux camarades fonctionner. Ces deux derniers sont certainement dans une incertitude, plutôt liée aux processus de calcul. Ce groupe s'apparente donc à un ensemble " éclaté » où chacun est resté dans une activité personnelle indépendante d'une dynamique commune.

\section{Discussion/conclusion}

Que retenir de ces différentes analyses? Que pouvons-nous extraire de la diversité de ces descriptions de travaux en groupe ? Ces analyses de l'activité d'élèves travaillant en groupe montrent des réalités coopératives différentes, invalidant l'hypothèse selon laquelle cette forme d'action conjointe conduit systématiquement les élèves à échanger autour de conflits socio-cognitifs. Elles décrivent plusieurs postures coopératives d'élèves, induites par la dynamique du groupe auquel ils appartiennent. Leur analyse conduit à des types différents et non exhaustifs d'organisation du travail en groupe : les coopérateurs express, les stratèges de l'évitement, les complexés, les majoritaires, les découragés et les éclatés. 
Tableau 1 : Synthèse des analyses de travaux en groupe. Table 1: Summary of group work analyses

\begin{tabular}{|c|c|}
\hline $\begin{array}{l}\text { Groupes } 1 \\
\text { Les coopérateurs } \\
\text { express }\end{array}$ & $\begin{array}{l}\text { Les élèves sont entrés dans l'étude de la situation-problème. Chacun participe } \\
\text { aux échanges. Plusieurs conflits socio-cognitifs se produisent et aboutissent à } \\
\text { une incertitude partagée. Ces conflits ne se sont produits que sur un temps } \\
\text { inférieur à } 1 \text { minute. }\end{array}$ \\
\hline $\begin{array}{l}\text { Groupes } 2 \\
\text { Les stratèges de } \\
\text { l'évitement }\end{array}$ & $\begin{array}{l}\text { Les élèves ne débattent pas. Certains restent uniquement centrés sur la } \\
\text { fonction qui leur incombe. Un seul conflit se produit, rapidement écarté par } \\
\text { l'établissement d'un consensus de ne pas approfondir ce désaccord. Le groupe } \\
\text { consacre l'essentiel du temps à échanger sur autre chose que l'étude du } \\
\text { problème. }\end{array}$ \\
\hline $\begin{array}{c}\text { Groupes } \mathbf{3} \\
\text { Les complexés }\end{array}$ & $\begin{array}{l}\text { Les élèves ne réfléchissent pas individuellement au problème. L'un d'entre } \\
\text { centralise les échanges mais une intervention de l'enseignante est nécessaire. } \\
\text { Des désaccords naissent de la différence de résultats, mais ils ne sont pas } \\
\text { exploités parce que le groupe a l'impression d'être en retard par rapport aux } \\
\text { autres groupes. Ils discutent alors d'autres sujets. }\end{array}$ \\
\hline $\begin{array}{c}\text { Groupes } 4 \\
\text { Les majoritaires }\end{array}$ & $\begin{array}{l}\text { Un élève s'occupe de l'animation du groupe. Tous participent, mais un seul } \\
\text { développe des arguments corrects. Les autres élèves, majoritaires, pensent } \\
\text { avoir raison et écartent cette idée divergente. Le premier s'efface des } \\
\text { échanges et le groupe évoque des sujets hors-problème. }\end{array}$ \\
\hline $\begin{array}{c}\text { Groupes } 5 \\
\text { Les découragés }\end{array}$ & $\begin{array}{l}\text { Un élève se charge de la distribution de la parole. Le groupe semble, tout au } \\
\text { long du travail en représentation. Les partenaires se disent toujours en accord } \\
\text { et ne manifestent pas une concentration importante sur la tâche : les idées } \\
\text { avancées ne font jamais l'objet d'approfondissements parce que les élèves se } \\
\text { pensent incompétents. }\end{array}$ \\
\hline $\begin{array}{l}\text { Groupes } 6 \\
\text { Les éclatés }\end{array}$ & $\begin{array}{l}\text { Deux élèves échangent sur le problème tandis que les autres sont inactifs ou } \\
\text { bavardent avec des membres d'autres groupes. Les premiers entrent en } \\
\text { conflit autour de leurs réflexions, mais les seconds semblent ne pas être } \\
\text { concernés par le problème. }\end{array}$ \\
\hline
\end{tabular}

69 La première constatation qu'il est possible d'effectuer est la diversité des formes prises par l'activité des élèves. Sur les dix-sept groupes étudiés, six fonctionnements différents ressortent. De plus, au regard des attendus cognitifs du travail en groupe (susciter du questionnement par l'émergence d'incertitude par les conflits sociocognitifs), il apparait que seulement un seul type de groupe («les coopérateurs express ») semble atteindre cet objectif. Dans le panel étudié, ce type correspond à seulement trois groupes sur les dix-sept observés. Pour les quatorze autres, soit quelques élèves sont dans le doute (« les éclatés »), soit aucun ne semble avoir effectué ce cheminement vers du questionnement ${ }^{8}$. Pour «les stratèges de l'évitement », c'est en raison d'un refus des désaccords, pour "les complexés » et «les découragés », ce serait par un déficit de sentiment d'efficacité personnelle (Bandura, 2003) et pour « les majoritaires ", c'est par une non-prise en compte du seul avis divergent. Ces choix d'élèves, révélés par leur activité, s'expliquent en partie parce que les conflits entre pairs ne bénéficient pas d'une bonne image dans nos cultures : ils sont souvent évités, par les élèves et les enseignants, soit par souci de bienveillance, soit par stratégie d'évitement des difficultés (Buchs et al., 2008).

À première lecture, il est possible de retenir de cette étude que le travail en groupe, dans ce contexte de collecte de données, ne semble pas riche pour accompagner le processus d'apprentissage. Certes, les élèves et les enseignants ont été confrontés à l'effet Hawthorne (Mialaret, 2004) lié à la présence de la caméra (plusieurs extraits ont été écartés pour cette raison) et nous retenons que tous les groupes ne sont pas habitués à travailler de la sorte. Mais il reste impossible d'affirmer qu'organiser des groupes d'élèves est toujours vecteur de conflits socio-cognitifs débouchant sur une ouverture aux savoirs scolaires. Il convient donc de reconnaitre que «le travail en groupe est loin d'être la condition suffisante pour que se produisent des conflits sociocognitifs» (Rey \& Carette, 2019, p.40). Toutefois, d'autres conclusions ont été formulées avec l'équipe des enseignants. 
71 Au sujet des consignes de démarrage, la répartition des responsabilités n'a pas été organisée de manière ritualisée, la consigne n'a pas toujours été complète (par exemple en Mathématiques concernant la possibilité d'utiliser la calculette), à la fin de son énoncé, elle n'a pas fait systématiquement l'objet de questions à poser et de reformulations (ce qui a soulevé plusieurs fois le problème de l'usage de termes spécifiques), le temps de travail individuel préalable n'a pas toujours été respecté, la durée de 10 minutes semble longue et les élèves se retrouvent vite dans des discussions hors sujet.

72 Pendant la phase de travail en groupe, plusieurs stratégies d'évitement de penser (Boimare, 2008) ont été repérées : manque d'association de tous les élèves, non prise en compte des avis divergents, dévalorisation par comparaison sociale, refus d'accepter les controverses et les confrontations d'idées. Souvent, on repère des stratégies d'évitement par consensus de complaisance (Hugon, 2003): par exemple «les majoritaires » qui pensent avoir fini parce qu'une majorité se dégage. Par peur du conflit, relationnel, socio-cognitif ou cognitif, des élèves en viennent à accepter d'être en accord avec l'idée dominante, la première formulée ou exprimée par le plus influent du groupe, ce qui leur permet de ne plus avoir à étudier le problème (puisqu'une solution a été acceptée).

73 Le recours à ces stratégies rappelle les différences existant entre les buts de maitrise et de performance (Darnon et al., 2006) : une orientation vers des buts de maitrise favorise une relation épistémique aux problèmes et aux conflits socio-cognitifs. Autrement dit, quand les élèves se fixent des buts de maitrise, ils cherchent à apprendre, pas seulement à exécuter une consigne ou à développer une posture d'évitement de cette consigne. L'adoption de buts de maitrise favorise la régulation épistémique alors que l'adoption de buts de performance prédit une régulation relationnelle, vis-à-vis des pairs (pour maintenir une réputation ou une reconnaissance ou éviter une mise à l'écart) ou vis-à-vis de l'enseignant (dans le cadre d'une relation éducative caractérisée par les phénomènes de projections qui la composent).

74 Ce travail permet donc la formulation de l'hypothèse selon laquelle il serait utile de former les élèves à ces réalités cognitives, en amont des situations de travail en groupe. Cette formation préalable des élèves leur permettrait d'être mieux avertis des écarts qui existent entre des buts de maitrise et des buts de performance, les premiers étant prioritaires dans les contextes scolaires. Elle aiderait donc à favoriser des postures de secondarisation (Bautier \& Goigoux, 2004) consistant à développer des habitudes pour déceler, derrière les tâches énoncées, les activités cognitives attendues. Cette formation pourrait donc prendre plusieurs formes :

- en début d'année scolaire dans le cadre de la présentation d'habitudes de travail par l'intermédiaire de séances de formations prévues à cet effet ${ }^{9}$,

- avant d'engager du travail en groupe avec les élèves, sous forme d'une ritualisation des attendus cognitifs (par exemple avec l'aide d'un document projeté devant la classe : «La priorité n'est pas de participer, mais d'apprendre : répondre au problème à partir de ce que l'on sait déjà, poser des questions, chercher à ne pas être d'accord avec les idées apportées par les autres, vérifier les idées et les réponses données, ne pas craindre l'incertitude... »),

- en bilan d'une séance comprenant du travail en groupe, pour permettre à chacun une expression relative aux attendus prioritaires de cette forme coopérative : l'engagement dans le problème, la réflexion individuelle, la découverte d'avis différents, la recherche de désaccords, la conscience d'un questionnement, des réponses obtenues à ces besoins). Ces 
bilans peuvent être ritualisés à l'aide d'outils comme le carré ou la toile d'évaluation (Connac, 2017b) et avoir pour effet, si besoin, la modification d'organisations de la mise au travail des élèves. en raison de flous relatifs à la finalisation de l'activité. Si cette finalisation devenait individuelle, après le travail en groupe et la formalisation et par une réalisation personnelle d'une consigne de mise en œuvre immédiate, les élèves pourraient être plus orientés vers des buts de maîtrise. De plus, cette consigne individuelle à réaliser avant la fin de la séance, validée par l'enseignant, pourrait conduire les élèves à disposer de rétroactions relatives à leurs premières compréhensions (Mercier-Brunel, 2017 ; Roediger, Putnam, \& Smith, 2011), que celles-ci soient correctes ou pas. Ils auraient alors la possibilité de basculer dans le circuit de la récompense (Favre, 2015) ou de disposer d'éléments pour faire de leurs erreurs des occasions pour mieux apprendre (Astolfi, 1997).

L'analyse de ces activités de travail en groupe conduit à la formulation de repères aprioriques facilitant leur recours au sein de situations d'enseignement-apprentissages. L'accrochage des élèves et leur enrôlement dans la tâche semblent facilités par une organisation intentionnée (tournée vers l'émergence de conflits d'idées et finalisée par une réalisation individuelle) et rigoureuse (avec une articulation individuel-groupecollectif, des règles de fonctionnement, des étapes ritualisées, des fonctions pour réduire la charge cognitive des interactions coopératives, une durée courte du travail en groupe). Il semble également peu pertinent d'obliger un élève qui ne le souhaite pas à participer en groupe, celui-ci adoptant la plupart du temps des postures de retrait l'empêchant d'entrer dans l'activité cognitive attendue (Connac, 2018c).

Il apparaît également nécessaire une acculturation des élèves aux conflits d'idées, pour qu'ils puissent résister aux tentations de consensus de complaisance et prioriser les buts de maitrise sur les buts de performance. Pour cela, en plus d'une formation initiale, une consigne explicite de valorisation des désaccords et un usage d'un artefact comme un placemat (Danquin \& Mattes, 2015) pourraient aider les élèves à comprendre ces logiques de travail scolaire. 
Figure 2 : Modèle de Placemat.

Figure 2: Placemat model

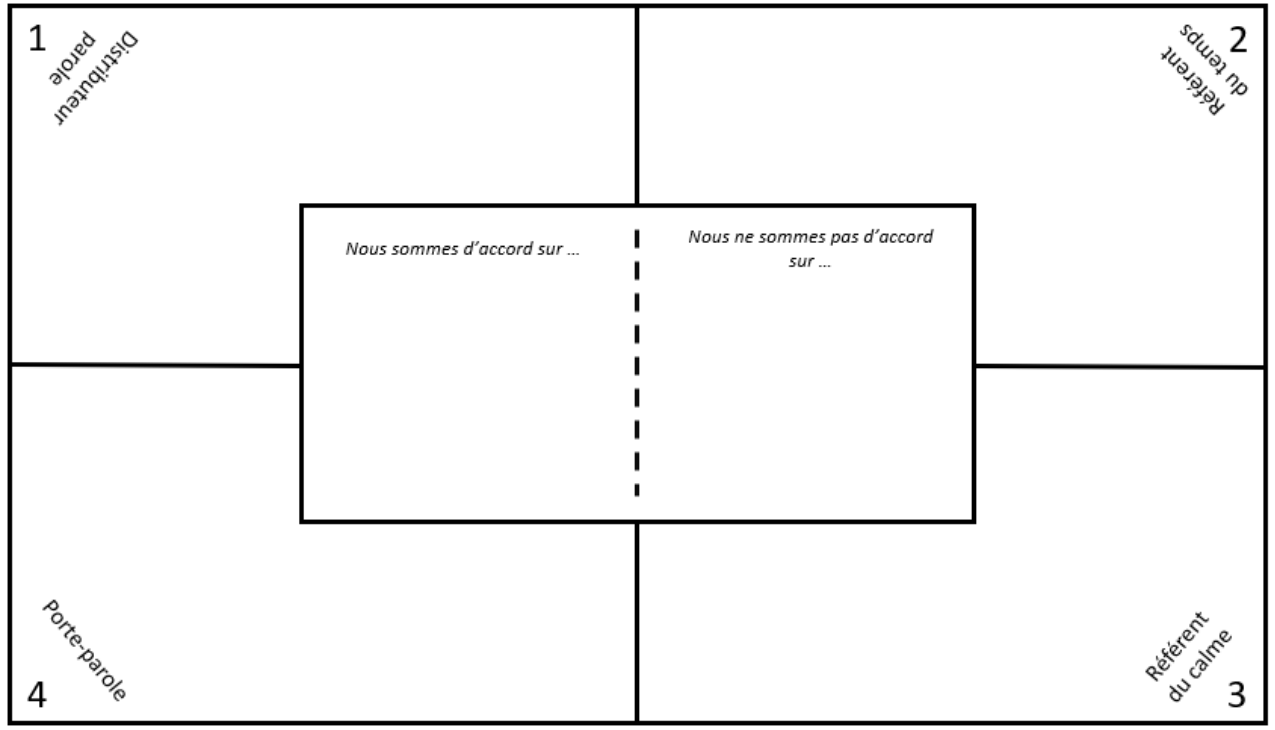

Enfin, l'étude de ces activités de travail en groupe souligne une nécessité de favoriser les conflits cognitifs après l'émergence des conflits socio-cognitifs permis par les interactions sociales entre pairs. Ce processus vers les apprentissages débute par les phénomènes successifs d'enrôlement dans la tâche (par une présentation lente et précautionneuse de la consigne, la phase préalable de travail individuel et les échanges autour de la compréhension de la consigne lors du travail en groupe). Il se poursuit par ce qui se produit au sein des groupes lors des moments de confrontations, qui, nous l'avons vu, ne sont pas majoritaires.

L'émergence des conflits cognitifs semble donc surtout émaner du traitement que l'enseignant organise des diverses idées produites avant le temps collectif d'exploitation de ces idées. Au regard des résultats indiqués par cette étude, ce serait principalement au moment où l'enseignant présente devant tous les élèves la diversité des réponses évoquées qu'apparaitrait du doute dans la conscience de la plupart d'entre eux. Ainsi, il semble que, pour aboutir à ce moment d'incertitude partagée, l'enseignant gagne à noter au tableau toutes les idées différentes émises par les groupes, explique pourquoi il en retire certaines en raison de leurs incohérences (souvent liées à une erreur de compréhension de la consigne ou de redondances) et mette en exergue les quelques propositions restantes qui créent du litige. Lorsque les élèves reconnaissent ne plus pouvoir trancher avec des arguments recevables, l'enseignant peut alors estimer qu'il s'agit d'un «kaïros », un moment opportun pour répondre aux questions qu'ils se posent. C'est donc par une posture de transmission des savoirs experts que les doutes pourraient progressivement disparaitre, pour laisser place soit à de nouvelles compréhensions soit à une validation de ce que certains savaient déjà et qu'ils ne parvenaient plus à justifier en raison des arguments qui leur étaient opposés et de l'incertitude de la situation.

En appui sur toutes ces conclusions, nous sommes en mesure de les synthétiser à travers une modélisation enrichie d'un dispositif en 12 préconisations successives pour l'organisation de travail en groupe d'élèves. 
En amont de la séance comportant du travail en groupe :

1. Former les élèves aux attendus cognitifs du travail en groupe (l'engagement dans la tâche, la confrontation d'idées et le passage nécessaire par de l'incertitude) ainsi qu'aux diverses étapes qui précèdent et suivent cette forme coopérative. Cette formation intervient en amont, au début et à la suite des situations de travail en groupe, pour insister sur les priorités et autoorganiser le groupe en fonction des manques repérés.

2. Centrer la tâche sur une situation-problème, caractérisée par la présence d'un obstacle intellectuel dépassable par l'intermédiaire du savoir à enseigner.

Avant et pendant le travail en groupe :

1. Réserver un temps collectif suffisant pour la présentation du problème par l'enseignant, jusqu'à ce que les élèves n'aient plus de question à poser et soient en capacité de travailler adéquatement en autonomie.

2. Constituer les groupes à partir d'élèves volontaires (les autres réalisant seul le même travail), avec une taille réduite de 4 ou 5 membres, sur la base de composition aléatoire (pour éviter les regroupements d'élèves faibles ou socialement discriminés).

3. Laisser les élèves réfléchir individuellement au problème, pendant 2-3 minutes, afin qu'ils puissent s'approprier la consigne et être en mesure d'apporter des idées au groupe (s'ils ont choisi de travailler avec d'autres).

4. Distribuer aléatoirement des rôles aux élèves pour indiquer clairement qui a la responsabilité de quoi et pour, de ce fait, réduire la charge supplémentaire causée par les activités transactives et pallier le retrait de l'enseignant.

5. Ne pas dépasser une durée du travail en groupe de 5 à 10 minutes, avec un rappel initial de la consigne d'échanger sur les désaccords et leur matérialisation à l'aide d'un artefact tel qu'un placemat.

Après le travail en groupe :

1. Conduire le temps de remontée des groupes pour mettre en évidence la palette la plus étendue des idées différentes et les organiser afin d'exacerber les incertitudes et le questionnement dans l'esprit des élèves.

2. Repérer le kaïros, un moment opportun où l'enseignant perçoit dans la classe l'atteinte d'une zone de crête entre trop d'incertitude qui pourrait décourager les plus fragiles et suffisamment d'attentes de réponses pour maintenir l'attention cognitive et compenser le doute dans lequel les élèves se trouvent.

3. Répondre aux questions que les élèves se posent en apportant les savoirs experts en mesure de dépasser l'obstacle porté par la situation-problème. Présenter ces savoirs le plus clairement possible et par l'intermédiaire de réactions et de questions spontanées.

En fin de séance :

1. Donner aux élèves un exercice d'application directe des savoirs transmis, afin que chacun ait la possibilité de tester sa perception de compréhension à l'épreuve d'une validation par l'enseignant, via une rétroaction immédiate.

2. Terminer la séance comprenant du travail en groupe par un rapide bilan ritualisé, accordant à chacun une expression individuelle concernant les effets de l'organisation du travail sur les buts de maitrise (Cf. étape 1).

Devant la non-évidence et le foisonnement de ces développements, il parait désormais flagrant qu'une telle structuration du travail coopératif des élèves nécessite une formation des enseignants: sur l'acte d'apprendre, la dimension sociale de l'apprentissage, les différentes déclinaisons de la coopération entre élèves, les spécificités du travail en groupe, les distinctions avec le travail en équipe et le travail de groupe, les étapes d'organisation, les principales précautions et les attentions 
pédagogiques prioritaires pour une telle conduite. Il semble opportun de penser cette formation en faisant vivre de manière récurrente du travail en groupe en formation, selon des logiques homomorphes (Develay, 1994), autour de situations-problèmes relatives à des enjeux de métiers et en se donnant du temps pour analyser les phénomènes cognitifs induits par cette forme de mise au travail.

Cette étude s'achève donc sur l'identification de nouvelles attentions concernant l'organisation du travail en groupe d'élèves. Celles-ci devraient objectiver le recours pédagogique au travail en groupe, en l'orientant principalement vers la valorisation des conflits cognitifs préparant l'appropriation de nouveaux savoirs. Des recherches futures devront approfondir dans ce sens l'observation de leurs activités, notamment pour affiner et compléter la typologie des postures coopératives d'élèves. Pour cela, nous envisageons de coupler les formes d'analyses effectuées à une approche phénoménologique qui récolte l'avis des élèves sur ce qu'ils viennent de réaliser.

\section{BIBLIOGRAPHIE}

Astolfi, J-P. (1992). Apprendre par franchissement d'obstacles? Repères, recherches en didactique du français langue maternelle, 5, 103-116.

Astolfi, J-P. (1997). L'erreur, un outil pour enseigner. Issy-les-Moulineaux : ESF Éditeur.

Astolfi, J-P. (2008). La saveur des savoirs. Disciplines et plaisir d'apprendre. Paris : ESF.

Audrin, C. (2020). Les émotions dans la formation enseignante : une perspective historique.

Recherches en éducation, 41, 5-19.

Bakhtine, M. (1978). Esthétique et théorie du roman. Paris : Gallimard.

Bandura, A. (2003). Auto-efficacité. Le sentiment d'efficacité personnelle. Paris : De Boeck Université.

Barbier, J.M. (2011). Vocabulaire d'analyse des activités. Paris : PUF.

Bardin, L. (1997). L'analyse de contenu. Paris : PUF.

Barthes, A., Lange, J.M., \& Tutiaux-Guillon, N. (2017). Dictionnaire critique des concepts des "éducations à ». Paris : L'Harmattan.

Bautier, E., Goigoux, R. (2004). Difficultés d'apprentissage, processus de secondarisation et pratiques enseignantes : une hypothèse relationnelle. Revue Française de Pédagogie, 148, 89-100.

Bécu-Robinault, K., \& Robin, O. (2017). Des consignes aux activités des élèves - une étude de cas sur les phses de la Lune en Cycle 3. Éducation \& Didactique [En ligne], 11-13, URL : http:// journals.openedition.org/educationdidactique/2826.

Blais, M.C., \& Fleury, M.A. (1996). Enseigner dans des classes hétérogènes la pédagogie interactive. CRESAS/INRP.

Blais, M., \& Laval, C. (2019). Neuroéducation - Le cerveau au centre de l'école. Paris : Tschann \& Cie. Boimare, S. (2008). Ces enfants empêchés de penser. Paris : Dunod. 
Brassard, N. (2012). Évaluation et rétroaction : comment en tirer profit ? Le Tableau, 1(4).

Brousseau, G. (1998). Théorie des situations didactiques. Grenoble : La Pensée Sauvage.

Bruner, J. S., (2011). Le développement de l'enfant, savoir-faire, savoir dire. Paris : PUF.

Buchs, C., Darnon, C., Quiamzade, A. Mugny, G., \& Butera, F. (2008). Conflits et apprentissage : régulation des conflits sociocognitifs et apprentissage. Revue française de pédagogie, 163, 105-125.

Caussidier, C. (2014). Le sujet neurocognitif et l'éducation : un paradoxe ? Éducation et Socialisation, 36, en ligne : https://journals.openedition.org/edso/979

CNESCO, (2017). Différenciation pédagogique-Comment adapter l'enseignement pour la réussite de tous les élèves? Paris : CNESCO/IFE.

Cole, A.L. (1989). Researcher and teacher: Partners in theory building. Journal of Education for Teaching, 75(3), 225-237.

Connac, S. (2017a). Enseigner sans exclure. Paris : ESF Sciences Humaines.

Connac, S. (2017b). La coopération entre élèves. Futuroscope: Éditions Canopé.

Connac, S. (2018a). Impact de la coopération entre élèves sur leurs performances scolaires en $6 \mathrm{e}$. Les Sciences de l'Éducation pour l'Ere Nouvelle, 51/4, 31-62.

Connac, S. (2018b). Neuroéducation et pédagogie. Éducation et Socialisation, 49, en ligne : https:// journals.openedition.org/edso/3556

Connac, S. (2018c). Ce que disent des élèves sur les classes coopératives en collège et lycée. Tréma, 50, en ligne : https://journals.openedition.org/trema/4265

Connac, S. (2020). La coopération, ça s'apprend. Paris : ESF Sciences Humaines.

Cottereau, D. (2007). Alterner pour apprendre - Entre pédagogie de projet et pédagogie de l'écoformation. Réseau École et Nature.

Danquin, R., \& Mattes, W. (2015). 52 méthodes pratiques pour enseigner. Futuroscope : Éditions Canopé.

Darnon, C., Buchs, C., \& Butera, F. (2006). Buts de performance et de maîtrise et interactions sociales entre étudiants : la situation particulière du désaccord avec autrui. Revue française de pédagogie, 155, 35-44.

De Vecchi, G. (2017). Former l'esprit critique - Pour une pensée libre. Paris : ESF Éditeur.

Desgagné, D., Bednarz, N., Lebuis, P., Poirier, L., \& Couture, C. (2001). L'approche collaborative de recherche en éducation : un rapport nouveau à établir entre recherche et formation. Revue des sciences de l'éducation, 27(1), 33-64.

Desgagné, S. (1997). Le concept de recherche collaborative : l'idée d'un rapprochement entre chercheurs universitaires et praticiens enseignants. Revue des sciences de l'éducation, 23(2), 371-393.

Develay, M. (1992). De l'apprentissage à l'enseignement. Paris : ESF Éditeur.

Develay, M. (1994). Peut-on former les enseignants ? Paris : ESF Éditeur.

Dieumegard, G. (2011). Dimensions cognitives et sociales dans l'étude de l'activité des élèves : La représentation comme inférence individuelle-sociale dans le cours d'expérience. Éducation \& didactique, 5(3), 33-60.

Doise, W., \& Mugny, G. (1981). Le développement social de l'intelligence. Paris : Interéditions. 
Engeström, Y. (1987). Learning by expanding. An activity-theorital approach to developmental research. Helsinki : Orienta-Konsultit.

Fabre, M., \& Vellas, E. (Eds.) (2006). Situations de formation et problématisation. Bruxelles : De Boeck Supérieur.

Favre, D. (2015). Cessons de démotiver les élèves. Paris : Dunod.

Filliettaz, L. \& Schubauer-Leoni, M. (2008). Les processus interactionnels dans leurs dimensions interpersonnelles, socio-historiques et sémiotiques. In L. Filliettaz (Ed), Processus interactionnels et situations éducatives (pp. 7-39). Louvain-la-Neuve, Belgique : De Boeck Supérieur. https://doi.org/ 10.3917/dbu.filli.2008.01.0007

Ginestié, J., \& Tricot, A. (Eds.) (2013). Activité d'élèves, activité d'enseignants en éducation scientifique et technologique. RDST, 8 , http://journals.openedition.org/rdst/755

Giordan, A. (2015). Les neurosciences, la grande illusion en education. Educavox, en ligne : https://www.educavox.fr/innovation/recherche/les-neurosciences-la-grande-illusion-eneducation

Glaser, B.G., \& Strauss, A.L. (2010). La découverte de la théorie ancrée - Stratégies pour la recherche qualitative. Paris : Armand Colin.

Huber, M. (1999). Apprendre en projets. Chronique Sociale.

Hugon, M.A. (2003). Vers une approche coopérative des apprentissages à l'école, en formation et dans la recherche pédagogique. Note de synthèse pour une HDR. Université Paris-Ouest Nanterre La Défense.

Kahn, S. (2010). Pédagogie Différenciée. Bruxelles : De Boeck.

Kirschner, P., Kirschner, F., Sweller, J., \& Zambrano, R. (2018). From cognitive load theory to collaborative cognitive load theory. International Journal of Computer-Supported Collaborative Learning, 13, 213-233.

Leblanc, S., \& Sauvaire-Maltrana, C. (2019). Comprendre l'activité d'enseignant(e)s utilisant des formes pédagogiques coopératives nouvelles dans le secondaire. Activités, 16(1), [En ligne]: http://journals.openedition.org/activites/3901

Léontiev, A. (1975). Activité, conscience, personnalité (Traduction 1984). Moscou : Éditions du Progrès.

Lieberman, A. (1986). Collaborative research: Working with, not working on... Educational Leadership, 43(5), 29-32.

Lorcerie, F. (2020). Hétérogénéité. Cahiers Pédagogiques, HSN 54, 34-35.

Magendie, É. (2009). L'activité réelle des élèves en EPS : entre prescriptions et préoccupations : études de cas d'élèves de 3ème en volley-ball. Thèse de doctorat en STAPS, Université de Bordeaux 2.

Magendie, É., \& Bouthier, D. (2012). Des ruptures de contrat au sens de l'activité pour les élèves : une approche clinique de l'activité réelle en EPS. Éducation \& didactique, 6(2), 27-46.

Marcel, J.F. (Ed.), (2015). La recherche-intervention par les sciences de l'éducation-Accompagner le changement. Dijon : Educagri éditions.

Masson, S. (2020). Activer ses neurones - Pour mieux apprendre et enseigner. Paris : Odile Jacob.

Mercier-Brunel, Y. (2017). Soutenir l'autorégulation des apprentissages en séance de correction collective. In S. Cartier, \& L. Mottier Lopez (Eds.), Soutien à l'apprentissage autorégulé en contexte scolaire: Perspectives francophones (pp. 137-158). Québec : Presses de l'Université du Québec. 
Meirieu, P. (1996). Itinéraire des pédagogies de groupe - Apprendre en groupe. Chronique Sociale.

Meirieu, P. (2016). Apprendre... oui, mais comment (Pédagogies références). Issy-les-Moulineaux : ESF éditeur.

Mialaret, G. (2004). Les méthodes de recherche en sciences de l'éducation. Paris : PUF.

Musial, M., \& Tricot, A. (2020). Précis d'ingénierie pédagogique. Bruxelles : De Boeck Supérieur.

Netter, J. (2018). Culture et inégalités à l'école. Esquisse d'un curriculum invisible. Rennes : Presses universitaires de Rennes.

Nonnon, É. (2008). Tensions et dynamique des interactions dans les échanges scolaires. In L. Filliettaz (Ed.), Processus interactionnels et situations éducatives (pp. 43-65). Louvain-la-Neuve, Belgique : De Boeck Supérieur. https://doi.org/10.3917/dbu.filli.2008.01.0043

Paillé, P., \& Mucchielli, A. (2012). L'analyse qualitative en sciences humaines et sociales. Paris : Armand Colin.

Pekrun, R. (2006). The Control-Value Theory of Achievement Emotions: Assumptions, Corollaries, and Implications for Educational Research and Practice. Educational Psychology Review, 18(4), 315-341.

Pekrun, R. (2014). Emotions and Learning. Educational Practices Series, 24, En ligne https:// unesdoc.unesco.org/ark:/48223/pf0000227679

Perret-Clermont, A.N. (1979). La construction de l'intelligence dans l'interaction sociale. Berne : Peter Lang.

Raab, R. (2014). Apprentissage en autonomie et stratégies d'évitement de l'obstacle. Questions Vives, 22, https://doi.org/10.4000/questionsvives.1653

Rey, B., \& Carette, V. (2019). Enseignement et apprentissage dans le secondaire - Un état des connaissances et des problèmes. Louvain-La-Neuve : Academia L'Harmattan.

Robbes, B. (2013). Épistémologies de la pédagogie, relations aux savoirs et à la didactique. Éducation et socialisation, 34 [En ligne], https://journals.openedition.org/edso/434

Robert, A., \& Hache, C. (2013). Pourquoi, comment comprendre ce qui se joue en classe de mathématiques? In F. Vandebrouck (Ed.), Activités des élèves et pratiques des enseignants en classe de mathématiques (pp. 25-86). IREM de Paris, Cahiers du Laboratoire de Didactique André Revuz, hal-02110844.

Roediger, H-L., Putnam, A-L., \& Smith, M-A. (2011). Ten Benefits of Testing and Their Applications to Educational Practice. Psychology of Learning and Motivation, 55, 1-36.

Rogalski, A. (2013). Théorie de l'activité et cadre de développementaux pour l'analyse liée des pratiques des enseignants et des apprentissages des élèves. In F. Vandebrouck (Ed.) Activités des élèves et pratiques des enseignants en classe de mathématiques (pp. 3- 24). IREM de Paris, Cahiers du Laboratoire de Didactique André Revuz, hal-02110844

Rousset, M. (2016). Neuroéducation, pourquoi ça ne prend pas? Le Monde. 8 décembre.

Sander, E., Gris, E., Gvozdic, K., \& Scheibling-Sève, C. (2018). Les neurosciences en éducation. Paris : Retz.

Sensevy, G., Messina, V., \& Lefeuvre, L. (2020). Enseignement constructiviste ou enseignement direct : il faut choisir. In Enseigner, ça s'apprend (pp. 49-68), Paris : Retz. 
Sensevy, G., \& Quilio, S. (2002). Les discours du professeur. Vers une pragmatique didactique. Revue française de pédagogie, 141, 47-56.

Sensevy, G., Santini, J., Cariou, D., \& Quilio, S. (2018). Preuves fondées sur la pratique, pratiques fondées sur la preuve : distinction et mise en synergie, Éducation et didactique, 12(2), 115-125.

Tiberghien, A., \& Vince, J. (2015). Étude de l'activité des élèves de lycée en situation d'enseignement de la physique. Cahiers du Français Contemporain, 10, 153-176.

Toullec-Théry, M. (2015). Des politiques françaises en matière d'éducation centrées sur l'individualisation, la personnalisation plus que sur le collectif: quels effets sur les apprentissages des élèves? Une contribution didactique. Paris : CNESCO.

Venturini, P. (2012). Action, activité, « agir » conjoints en didactique : discussion théorique. Éducation \& didactique, 6(1), 127-136. https://doi.org/10.4000/educationdidactique.1348 Veyrunes, P., \& Saury, J. (2009). Stabilité et auto-organisation de l'activité collective en classe : exemple d'un cours dialogué à l'école primaire. Revue française de pédagogie, 169(4), 67-76.

Zask, J. (2011). Participer : essai sur les formes démocratiques de la participation. Latresne : le Bord de l'eau.

\section{NOTES}

1. Une représentation peut se définir comme un contenu de la pensée, sous forme d'idées, d'opinions, de croyances, de savoirs sans distinction, souvent dans le sens d'une simplification parce qu'en lien avec le système de pensée de la personne. C'est « la manière dont un individu donné, à un moment donné, dans une situation donnée, mobilise ses connaissances antérieures » (Develay, 1992, p. 78).

2. Chez Leontiev, le but se rapporte à l'action et l'activité à des motifs.

3. Lorsqu'un élève a du mal à répondre à une question du professeur, celui-ci peut l'aider à trouver la réponse. Il peut l'orienter mais tomber aussi dans le piège de trop dire. En voulant aider, l'effet Topaze «souffle» la réponse. (Connac, 2017a ; Sensevy, Santini, Cariou, \& Quilio, 2018).

4. Il laisse donc délibérément de côté l'étude d'autres formes coopératives évoquées plus haut comme le travail en équipe, le travail en atelier, les fonctionnements en ilots, l'aide, l'entraide, le tutorat, les conseils coopératifs, les discussions démocratiques, les jeux coopératifs...

5. Ont participé à cette recherche Stéphanie Bréau, Alexandra Canézi, Lucie Chanu, Sylvain Connac, Guillaume Descourtis, Claire Dotto, Amélie Dumont, Delphine Dupré, Catherine Landry, Loïc Lefevre, Émilie Lhuillier, Jean-Marie Pinéro, Thierry Ponchaut, Carmen Rusu, Aurélie Suteau, Joëlle Tarayre.

6. Par l'intermédiaire d'un document collaboratif Framapad.

7. «On appelle "effet Hawthorne" les résultats positifs ou négatifs qui ne sont pas dus aux facteurs expérimentaux, mais à l'effet psychologique que la conscience de participer à une recherche ou d'être l'objet d'une attention spéciale exerce sur le sujet ou sur le groupe expérimental » (Mialaret, 2004, p. 110).

8. Groupes $1: \mathrm{n}=3$; Groupes $2: \mathrm{n}=4$; Groupes $3: \mathrm{n}=2$; Groupes $4: \mathrm{n}=1$; Groupes $5: \mathrm{n}=2$; Groupes $6: n=5$.

9. Par exemple, le défi « squelette », pour identifier les gestes nécessaires au travail en groupe ; le défi «Léonard», pour comprendre l'impact d'un conflit socio-cognitif pour apprendre; le défi «Dopamine», pour situer l'importance de l'incertitude et du doute dans un processus d'apprentissage (Connac, 2020). 


\section{RÉSUMÉS}

Les enseignants mettent régulièrement leurs élèves en travail en groupe. Il s'agit d'une pratique coopérative qui cherche à engager des apprentissages par la confrontation des idées, sous forme de conflits socio-cognitifs. Ces désaccords sont espérés parce qu'ils conduisent à des doutes utiles pour faciliter ensuite le rapport au savoir. Cette recherche s'est intéressée à l'activité de lycéens qui, dans des disciplines différentes, ont reçu pour consigne l'étude d'une situation-problème au sein de petits groupes. L'analyse des verbatims vidéo montre des réalités à chaque fois différentes. Rarement, les groupes ont conduit les élèves à se questionner et plusieurs stratégies d'évitement ont été repérées. Il ressort de ces mises en évidence une série de nouvelles hypothèses pour l'organisation d'un travail en groupe qui oriente les élèves vers des buts de maitrise et l'acceptation d'incertitudes.

Teachers frequently put their students to work in group situations. This is a cooperative practice that seeks to initiate learning through the confrontation of ideas, in the form of socio-cognitive conflicts. These disagreements are hoped for, because they lead to constructive doubts which will then facilitate our relationship with knowledge. This research focuses on the activity of high school students from different disciplines who were instructed to study a problem situation in small groups. The analysis of video verbatims shows different realities each time. The groups rarely guided the students to question themselves and several avoidance strategies were identified. These identifications reveal a series of new hypotheses for the organization of group work that leads students to master goals and accept doubts.

\section{INDEX}

Mots-clés : activité, conflit cognitif, conflit socio-cognitif, coopération, enrôlement, travail en groupe

Keywords : activity, cognitive conflict, socio-cognitive conflict, cooperation, enrollment, group work

\section{AUTEURS}

\section{SYLVAIN CONNAC}

Université Paul-Valéry de Montpellier - LIRDEF - sylvain.connac@univ-montp3.fr

\section{CARMEN RUSU}

Université Paul-Valéry de Montpellier - carmen-emanuela.rusu@univ-montp3.fr 